\title{
THE BOUNDARY BEHAVIOR OF A COMPOSITE MATERIAL
}

\author{
Maria NeUss-RADU ${ }^{1}$
}

\begin{abstract}
In this paper, we study how solutions to elliptic problems with periodically oscillating coefficients behave in the neighborhood of the boundary of a domain. We extend the results known for flat boundaries to domains with curved boundaries in the case of a layered medium. This is done by generalizing the notion of boundary layer and by defining boundary correctors which lead to an approximation of order $\varepsilon$ in the energy norm.

Résumé. On étudie ici le comportement au voisinage de la frontière du domaine de solutions de problèmes elliptiques à coefficients oscillant périodiquement. Les résultats, connus pour des frontières plannes, sont étendus au cas de frontières courbes et pour un milieu stratifié. On généralise pour cela la notion de couche limite et on définit des correcteurs de frontière qui conduisent à une approximation d'ordre $\varepsilon$ dans la norme énergie.
\end{abstract}

Mathematics Subject Classification. 35B27, 35B40.

Received: March 7, 2000. Revised: January 16, 2001.

\section{INTRODUCTION}

Various thermal and electrical conductivity problems in a periodic composite are modeled on the microscopic level by partial differential equations with periodically oscillating coefficients of the following type:

$$
\text { Find } u^{\varepsilon} \in H_{0}^{1}(\Omega): \quad-\partial_{i}\left(\mathcal{A}_{i j}^{\varepsilon}(x) \partial_{j} u^{\varepsilon}(x)\right)=f(x), \forall x \in \Omega \text {, }
$$

where $\Omega \subset \mathbb{R}^{n}$ is a bounded domain, $f \in L_{2}(\Omega)$, and $\varepsilon>0$ represents the period of the oscillations in the coefficients. The coefficient matrix $\mathcal{A}^{\varepsilon}$ describing the conductivity tensor of the material is given by:

$$
\mathcal{A}^{\varepsilon}(x):=\mathcal{A}\left(\frac{x}{\varepsilon}\right)
$$

where the matrix $\mathcal{A}(y)$ is defined as follows:

(i) The elements $\mathcal{A}_{i j}$ are periodic with period 1 ; i.e., $\mathcal{A}_{i j}$ are defined on the unit cube $Y=[0,1]^{n}$ and extended by periodicity over the whole space $\mathbb{R}^{n}$,

(ii) $\mathcal{A}_{i j}=\mathcal{A}_{j i}$

(iii) $\mathcal{A}$ is bounded and strongly elliptic; i.e., there exist $0<\lambda \leq \Lambda<+\infty$ such that, for a.e. $y \in Y$,

$$
\lambda|\eta|^{2} \leq \mathcal{A}_{i j}(y) \eta_{i} \eta_{j} \leq \Lambda|\eta|^{2}, \quad \forall \eta \in \mathbb{R}^{n} .
$$

We are interested in the behavior of the solution $u^{\varepsilon}$ for small values of the parameter $\varepsilon$. In this case the numerical treatment of this microscopic model is very difficult, often even impossible. In applications, however,

\footnotetext{
Keywords and phrases. Homogenization, generalized boundary layers, energy error estimates.

1 Institut für Angewandte Mathematik, Im Neuenheimer Feld 294, 69120 Heidelberg, Germany.

e-mail: Maria.Neuss-Radu@iwr.uni-heidelberg.de
} 
one is usually not interested in what exactly happens on the microscopic scale; one rather needs macroscopic (effective) approximations which take into account the local effects. In order to find such approximations, we look for the limit of $u^{\varepsilon}$ for $\varepsilon \rightarrow 0$ in properly chosen function spaces. This approach is called homogenization.

There are essentially two methods used to get the equations for the macroscopic approximations. The first one is the method of asymptotic expansions, see [8,21], or [19], and consists of two steps: the formal derivation of the homogenized equation and then the rigorous proof of the convergence of the microsolutions to the solution of the derived equation. The second method introduced by Nguetseng and Allaire in [18], resp. [3], replaces the two steps with a single step by using a new type of convergence called two-scale-convergence.

The macroscopic (homogenized) problem corresponding to $\left(P^{\varepsilon}\right)$ is the following boundary value problem with constant coefficients:

$$
\text { Find } u^{0} \in H_{0}^{1}(\Omega): \quad-\partial_{i}\left(\mathcal{A}_{i j}^{0} \partial_{j} u^{0}(x)\right)=f(x), \forall x \in \Omega
$$

where the homogenized coefficients $\mathcal{A}_{i k}^{0}$ representing the tensor of effective conductivity of the composite are given by

$$
\mathcal{A}_{i k}^{0}:=\left\langle\mathcal{A}_{i j}\left(\delta_{j k}+\partial_{y_{j}} w^{k}\right)\right\rangle_{Y}=\left\langle\left(\delta_{i l}+\partial_{y_{l}} w^{i}\right) \mathcal{A}_{l j}\left(\delta_{j k}+\partial_{y_{j}} w^{k}\right)\right\rangle_{Y} .
$$

Here and in the following, $\langle.\rangle_{Y}$ denotes the mean value operator over $\mathrm{Y}$. The functions $w^{k}, k=1, \ldots, n$ used in the definition of the homogenized coefficients are defined on the cell $Y=[0,1]^{n}$ and are the solutions to the so-called cell-problems:

$$
\text { Find } w^{k} \in H_{\mathrm{per}}^{1}(Y): \quad \partial_{y_{i}}\left(\mathcal{A}_{i j}(y)\left(\partial_{y_{j}} w^{k}(y)+\delta_{j k}\right)\right)=0, \forall y \in Y
$$

The function space $H_{\mathrm{per}}^{1}(Y)$ is the completion of the space $C_{\mathrm{per}}^{\infty}(Y)$ of infinitely differentiable, periodic functions on the cell $Y=[0,1]^{n}$ with respect to the $H^{1}$-norm.

Concerning the convergence of the solutions $u^{\varepsilon}$ to $u^{0}$ for $\varepsilon \rightarrow 0$, the following theorem holds:

Theorem 1.1. The sequence $u^{\varepsilon}$ converges to $u^{0}$ strongly in $L_{2}(\Omega)$, weakly in $H_{0}^{1}(\Omega)$ and in the two-scale sense.

Proof. See for example [3].

Now the question arises whether we have strong convergence of $u^{\varepsilon}$ towards $u^{0}$ in $H_{0}^{1}(\Omega)$. In general, this is not the case, i.e., the derivatives of $u^{\varepsilon}$ do not converge strongly in $L_{2}$, although they converge weakly. To improve the convergence, one needs a corrector.

Theorem 1.2. Using the solutions $w^{k}$ of the cell-problems, we can construct the approximation

$$
u_{\varepsilon}^{1}=u^{0}+\varepsilon \partial_{k} u^{0} w^{k}\left(\frac{\dot{\varepsilon}}{\varepsilon}\right)
$$

with the property that $u^{\varepsilon}-u_{\varepsilon}^{1}$ converges to zero in $H^{1}(\Omega)$ strongly.

Proof. See [6] or [8].

The study of the accuracy of the approximation $u_{\varepsilon}^{1}$ given in (2) is done by looking for estimates on the rate of convergence. To get such estimates, we have to treat separately the cases when the boundary $\partial \Omega$ of the domain $\Omega$ is empty or not. In the first case, which includes the situations when $\Omega=\mathbb{R}^{n}$ or when we have periodic boundary conditions on $\partial \Omega$, the approximation $u_{\varepsilon}^{1}$ obtained by using the homogenization techniques leads to an estimate of order $\varepsilon$ for the energy error, see [5]. In the second case, the error estimate in the energy norm is only of order $\varepsilon^{\frac{1}{2}}$ and can be found in $[6,13]$. This is due to the fact that the periodic structure of the approximation $u_{\varepsilon}^{1}$ is not consistent with the homogeneous Dirichlet boundary condition for the solution $u^{\varepsilon}$. In order to catch the boundary effects on the solution $u^{\varepsilon}$, we have to improve this approximation by adding a 
corrector. A possible choice for this corrector is given in the following theorem. However, in order to be able to deal with problems on unbounded domains, we first have to introduce several further function spaces.

Consider a domain $\Omega$ bounded or unbounded, and let $C^{\infty}(\bar{\Omega})$ be the space of all infinitely differentiable functions on $\Omega$ such that all derivatives have continuous extensions to $\partial \Omega$. We define the space $\dot{H}^{1}(\Omega)$ as the closure of

in the norm

$$
\left\{u \in C^{\infty}(\bar{\Omega}) / \mathbb{R}:\|\nabla u\|_{L_{2}(\Omega)} \leq C\right\}
$$

$$
\|u\|_{\dot{H}^{1}(\Omega)}:=\|\nabla u\|_{L_{2}(\Omega)} .
$$

We also introduce the function space $\dot{H}_{0}^{1}(\Omega)$ as the subspace of $\dot{H}^{1}(\Omega)$ consisting of functions with vanishing trace on $\partial \Omega$.

Theorem 1.3. Let $\Omega$ be a bounded or unbounded domain with Lipschitz-boundary. For $\Omega$ bounded, let $u^{\varepsilon}$ and $u^{0}$ be the solutions of $\left(P^{\varepsilon}\right)$, respectively $\left(P^{0}\right)$, with $f \in L_{2}(\Omega)$. For $\Omega$ unbounded and $f \in C_{0}^{\infty}(\bar{\Omega})$, we search for solutions $u^{\varepsilon}$ and $u^{0}$ lying in $\dot{H}_{0}^{1}(\Omega)$.

Further, let $v^{\varepsilon}$ be the solution of the problem:

$$
\begin{array}{cc}
-\partial_{i}\left(\mathcal{A}_{i j}^{\varepsilon}(x) \partial_{j} v^{\varepsilon}(x)\right)=0 & \text { for } x \in \Omega, \\
v^{\varepsilon}(x)=-\varepsilon \partial_{k} u^{0}(x) w^{k}\left(\frac{x}{\varepsilon}\right) & \text { for } x \in \partial \Omega .
\end{array}
$$

If the homogenized solution $u^{0}$ satisfies the condition $\left\|\nabla^{2} u^{0}\right\|_{L_{2}(\Omega)} \leq C$, and the cell-solutions $w^{k} \in W^{1, \infty}(Y)$, then we have the estimate

$$
\left\|u^{\varepsilon}-\left(u^{0}+\varepsilon \partial_{k} u^{0} w^{k}\left(\frac{\dot{\varepsilon}}{\varepsilon}\right)+v^{\varepsilon}\right)\right\|_{\dot{H}^{1}(\Omega)} \leq C \varepsilon\left\|\nabla^{2} u^{0}\right\|_{L_{2}(\Omega)} .
$$

Proof. For bounded domains, the result has been proven in $[15,16]$. For unbounded domains see the Appendix in [17].

The drawback of the corrector $v^{\varepsilon}$ given in Theorem 1.3 is that it is given only implicitly, and that we do not know anything about its decay. Thus, the numerical computations for this corrector would have the same complexity as the computation for the initial solution $u^{\varepsilon}$. To avoid this, we would like to have correctors whose energies concentrate in a neighborhood of the boundary of the domain. So far, such boundary correctors could be defined only for the situations when the domain is a half space whose boundary intersects the axes of periodicity in an angle with rational slope, see $[6,7,9,12,14,20]$, when it is an infinite strip with the same property, see [19], or a rectangular domain, see $[4,10]$. For these domains, the boundary corrector can be constructed with the help of special functions called boundary layers. They are solutions to elliptic problems defined on a semi-infinite strip $[0,1]^{n-1} \times[0, \infty]$, periodic in $y_{1}, \ldots, y_{n-1}$, decaying exponentially with respect to $y_{n}$.

For geometries other than the ones mentioned above, the construction of the classical boundary layers is not possible and, to our knowledge, there are no results concerning boundary correctors for general (say smooth) domains.

In our paper, we generalize the notion of boundary layer and define boundary correctors for general smooth domains. After restricting to a special class of domains, in the case of a layered medium, we prove that the defined boundary corrector is weakly differentiable with respect to the boundary parameters and that it leads to an error estimate of order $\varepsilon$ with respect to the energy norm.

The paper is organized as follows: In Section 2, we recall the techniques used for the construction of the boundary correctors for the half space and give a new proof for the $\varepsilon$-estimate in the energy norm. In Section 3 , we generalize the notion of boundary layer in the sense that we define boundary layers corresponding to each point of the boundary $\partial \Omega$, and that we drop the periodicity assumptions for the boundary layers. Using these generalized boundary layers and a tubular neighborhood of $\partial \Omega$, we define boundary correctors for general 
smooth domains. In order to prove weak differentiability of the boundary corrector with respect to the boundary parameters, in Section 4 we restrict to laminate composites and domains of the form:

$$
\Omega=\left\{x=\left(x_{1}, \ldots, x_{n}\right) \in \mathbb{R}^{n} \mid\left(x_{1}, \ldots, x_{n-1}\right) \in \mathbb{R}^{n-1}, x_{n}>g\left(x_{1}, \ldots, x_{n-1}\right)\right\},
$$

where $g \in C^{2}\left(\mathbb{R}^{n-1}\right)$ satisfies $\|g\|_{C^{2}\left(\mathbb{R}^{n-1}\right)} \leq G<\infty$. For this situation, we prove an error estimate of order $\varepsilon$ with respect to the energy norm.

\section{An estimate OF ORDER $\varepsilon$ FOR THE HALF SPACE}

In this section, we consider the problem:

Find $u^{\varepsilon} \in \dot{H}_{0}^{1}(\Omega)$, such that

$$
\int_{\Omega} \mathcal{A}_{i j}^{\varepsilon}(x) \partial_{j} u^{\varepsilon}(x) \partial_{i} \varphi(x) \mathrm{d} x=\int_{\Omega} f(x) \varphi(x) \mathrm{d} x, \forall \varphi \in \dot{H}_{0}^{1}(\Omega)
$$

on the half space $\Omega=\left\{x \in \mathbb{R}^{n}, x_{n}>0\right\}$ with $f \in C_{0}^{\infty}(\bar{\Omega})$. A boundary corrector for this problem was constructed for example in $[6,7,9,19]$, where we can also find energy estimates of order $\varepsilon$ for the error between $u^{\varepsilon}$ and the approximation given by help of the boundary corrector. In this section, we will prove a similar estimate by using a new approach. Our approach has two advantages: less regularity is required for the homogenized solution $u^{0}$, and - which is very important - it can also be used for domains with curved boundaries, as we will show in Section 4.2 .

We will now briefly recall some existence and regularity results concerning the boundary layers for the half space.

Definition 2.1. Let the $\mathcal{A}_{i j}$ satisfy the conditions (i), (ii), (iii). The boundary layers are the solutions $w^{b l, k}, k=1, \ldots, n$ of the problems

Find $w^{b l, k}$ such that:

$$
\begin{aligned}
& -\partial_{y_{i}}\left(\mathcal{A}_{i j}(y) \partial_{y_{j}} w^{b l, k}(y)\right)=0, \forall y \in Z \\
& w^{b l, k}\left(y^{\prime}, 0\right)=-w^{k}\left(y^{\prime}, 0\right), \forall y^{\prime} \in Z^{\prime} \\
& w^{b l, k} \text { is periodic in } y^{\prime} \\
& \nabla w^{b l, k} \text { decreases exponentially as } y_{n} \rightarrow \infty .
\end{aligned}
$$

where $\left.Z=Z^{\prime} \times\right] 0, \infty\left[\right.$ is called the boundary layer cell and $\left.Z^{\prime}=\right] 0,1\left[^{n-1}\right.$ (see Fig. 1).

To give the variational formulation of the boundary layer problems and also a rigorous formulation of the condition on the decay, we introduce the following weighted spaces, see [14]. For $\gamma>0$, set

$$
W_{\gamma}(Z)=\left\{w \in L_{2}(] 0, R\left[, H_{\mathrm{per}}^{1}\left(Z^{\prime}\right)\right), \forall R<\infty, \mathrm{e}^{\gamma y_{n}} \nabla w \in L_{2}(Z)\right\}
$$

and

$$
W_{\gamma}^{0}(Z)=\left\{w \in W_{\gamma}(Z), w\left(y^{\prime}, 0\right)=0\right\} .
$$

They are Hilbert spaces for the scalar product given by:

$$
\langle v, w\rangle_{W_{\gamma}(Z)}=\int_{Z} \mathrm{e}^{2 \gamma y_{n}} \nabla v \nabla w \mathrm{~d} y .
$$

This scalar product then induces the norm

$$
\|w\|_{W_{\gamma}(Z)}=\left\|\mathrm{e}^{\gamma y_{n}} \nabla w\right\|_{L_{2}(Z)}
$$




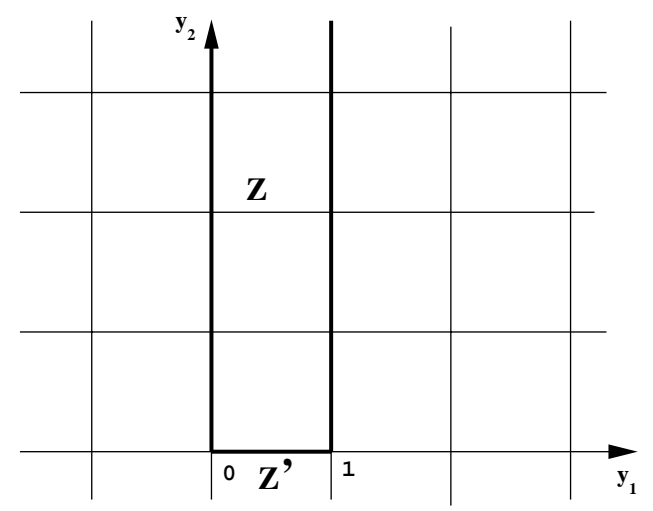

FiguRE 1. The boundary layer cell Z.

Let now $\hat{w}^{k}$ be an extension in $W_{\gamma}(Z)$ of the boundary values $\left.w^{k}\right|_{Z^{\prime}}$ to the whole boundary layer cell Z. Then the variational formulation of the boundary layer problems is:

Find $w^{b l, k}+\hat{w}^{k} \in W_{\gamma}^{0}(Z):$

$$
\int_{Z} \mathcal{A}_{i j}(y) \partial_{y_{j}} w^{b l, k}(y) \partial_{y_{i}} \varphi(y) \mathrm{d} y=0, \quad \forall \varphi \in W_{\gamma}^{0}(Z)
$$

The following theorem gives the existence and uniqueness of the $w^{b l, k}$ in $W_{\gamma}(Z)$.

Theorem 2.2. Let $\rho_{1}$ be the smallest nonzero eigenvalue of the Laplace-operator on $Z^{\prime}$ for periodic boundary conditions. Then for every $0<\gamma<\frac{\lambda \sqrt{\rho_{1}}}{2 \Lambda}$ there exist solutions $w^{b l, k}$ to the boundary layer-problems (7), and they are uniquely determined in $W_{\gamma}(Z)$. The following estimates hold:

$$
\left\|w^{b l, k}\right\|_{W_{\gamma}(Z)} \leq C\left\|\hat{w}^{k}\right\|_{W_{\gamma}(Z)} .
$$

Proof. This theorem is a more precise formulation of the result given in Theorem 10.1 from [14].

Let us now refer to some regularity properties of the $w^{b l, k}$.

Theorem 2.3. Let the coefficients $\mathcal{A}_{i j} \in C^{0, \kappa}\left(\mathbb{R}^{n}\right), 0<\kappa<1$. Then for $y^{0} \in Z$ and $R>0$ with $y_{2}^{0} \geq 4 R$ we have that $w^{b l, k} \in C^{1, \kappa}\left(\bar{B}_{4 R}\left(y^{0}\right)\right)$ for $k=1, \ldots, n$, and the following estimates hold:

$$
\left\|\nabla w^{b l, k}\right\|_{C^{0, \kappa}\left(\bar{B}_{R}\left(y^{0}\right)\right)} \leq C(R) \mathrm{e}^{-\gamma y_{2}^{0}}\left\|w^{b l, k}\right\|_{W_{\gamma}(Z)} .
$$

Proof. Without loss of generality, we can assume that $B_{4 R}\left(y^{0}\right) \subset Z$. Then $w^{b l, k} \in H_{2}^{1}\left(B_{4 R}\left(y^{0}\right)\right)$ is a solution of the equation

$$
\partial_{y_{i}}\left(\mathcal{A}_{i j}(y) \partial_{y_{j}} w^{b l, k}(y)\right)=0
$$

in $B_{4 R}\left(y^{0}\right)$. Then standard regularity results imply that $w^{b l, k} \in C^{1, \kappa}\left(\bar{B}_{4 R}\left(y^{0}\right)\right)$, and when we set

$$
\bar{w}^{b l, k}=\frac{1}{\left|B_{4 R}\left(y^{0}\right)\right|} \int_{B_{4 R}\left(y^{0}\right)} w^{b l, k}(y) \mathrm{d} y
$$

we have the estimate:

$$
\left\|w^{b l, k}-\bar{w}^{b l, k}\right\|_{C^{1, \kappa}\left(\bar{B}_{R}\left(y^{0}\right)\right)} \leq C(R)\left\|w^{b l, k}-\bar{w}^{b l, k}\right\|_{L_{2}\left(B_{4 R}\left(y^{0}\right)\right)} .
$$


Now, the Poincaré inequality on $B_{4 R}\left(y^{0}\right)$ yields:

$$
\begin{aligned}
\left\|\nabla w^{b l, k}\right\|_{C^{0, \kappa}\left(\bar{B}_{R}\left(y^{0}\right)\right)} & \leq C(R)\left\|\nabla w^{b l, k}\right\|_{L_{2}\left(B_{4 R}\left(y^{0}\right)\right)} \\
& =C(R) \mathrm{e}^{-\gamma\left(y_{2}^{0}-4 R\right)}\left(\int_{B_{4 R}\left(y^{0}\right)} \mathrm{e}^{2 \gamma\left(y_{2}^{0}-4 R\right)}\left|\nabla w^{b l, k}\right|^{2}\right)^{\frac{1}{2}} \\
& \leq C(R) \mathrm{e}^{-\gamma y_{2}^{0}}\left(\int_{B_{4 R}\left(y^{0}\right)} \mathrm{e}^{2 \gamma y_{2}}\left|\nabla w^{b l, k}\right|^{2}\right)^{\frac{1}{2}} \\
& \leq C(R) \mathrm{e}^{-\gamma y_{2}^{0}}\left\|w^{b l, k}\right\|_{W_{\gamma}(Z)} .
\end{aligned}
$$

Theorem 2.4. Let the coefficients $\mathcal{A}_{i j} \in C^{0, \kappa}\left(\mathbb{R}^{n}\right), 0<\kappa<1$. Let $y_{0} \in Z^{\prime}$ and $R>0$. We define:

$$
B_{R}^{+}\left(y_{0}\right)=B_{R}\left(y_{0}\right) \cap\left\{y_{n}>0\right\}
$$

and

$$
T_{R}\left(y_{0}\right)=B_{R}\left(y_{0}\right) \cap\left\{y_{n}=0\right\} .
$$

Then $w^{b l, k} \in C^{1, \kappa}\left(B_{4 R}^{+}\left(y_{0}\right) \cup T_{4 R}\left(y_{0}\right)\right)$ and

$$
\left\|\nabla w^{b l, k}\right\|_{C^{0, \kappa}\left(\bar{B}_{R}^{+}\left(y^{0}\right)\right)} \leq C(R)\left(\left\|w^{b l, k}\right\|_{W_{\gamma}(Z)}+\left\|w^{k}\right\|_{C^{1, \kappa}(\bar{Y})}\right) .
$$

Proof. This theorem can be proven in analogy to Theorem 2.3 .

Using the boundary layers $w^{b l, k}$ given by Definition 2.1, we now define the boundary corrector $w^{\varepsilon}$ by:

$$
w^{\varepsilon}(x)=\varepsilon \partial_{k} u^{0}(x) w^{b l, k}\left(\frac{x}{\varepsilon}\right), \quad \text { for all } \quad x \in \Omega,
$$

where $u^{0}$ is now the solution of the homogenized problem:

Find $u^{0} \in \dot{H}_{0}^{1}(\Omega)$, such that

$$
\int_{\Omega} \mathcal{A}_{i j}^{0} \partial_{j} u^{0}(x) \partial_{i} \varphi(x) \mathrm{d} x=\int_{\Omega} f(x) \varphi(x) \mathrm{d} x, \forall \varphi \in \dot{H}_{0}^{1}(\Omega) .
$$

Adding this boundary corrector to the approximation $u_{\varepsilon}^{1}$ given in (2), we obtain the following approximation for the solution $u^{\varepsilon}$ :

$$
u_{\varepsilon}^{a p p}(x):=u^{0}(x)+\varepsilon \partial_{k} u^{0}(x) w^{k}\left(\frac{x}{\varepsilon}\right)+w^{\varepsilon}(x) .
$$


This approximation leads to an energy-estimate of order $\varepsilon$ :

Theorem 2.5. If $u^{0}$ satisfies $\left\|\nabla^{2} u^{0}\right\|_{L_{2}(\Omega)} \leq C$ and the coefficients satisfy the additional assumption $\mathcal{A}_{i j} \in$ $C^{0, \kappa}\left(\mathbb{R}^{n}\right)$ with $0<\kappa<1$, then the following estimate holds:

$$
\left\|u^{\varepsilon}-\left(u^{0}+\varepsilon \partial_{k} u^{0} w^{k}\left(\frac{\dot{-}}{\varepsilon}\right)+w^{\varepsilon}\right)\right\|_{\dot{H}^{1}(\Omega)} \leq C \varepsilon\left\|\nabla^{2} u^{0}\right\|_{L_{2}(\Omega)} .
$$

Proof. Using the fact that $u^{\varepsilon}$ and $u^{0}$ satisfy the equations (5), respectively (12), we have for all $\varphi \in \dot{H}_{0}^{1}(\Omega)$ :

$$
\begin{aligned}
\int_{\Omega} \mathcal{A}_{i j}^{\varepsilon}(x) \partial_{j} & \left(u^{\varepsilon}(x)-u^{0}(x)-\varepsilon \partial_{k} u^{0}(x)\left(w^{k}\left(\frac{x}{\varepsilon}\right)+w^{b l, k}\left(\frac{x}{\varepsilon}\right)\right)\right) \partial_{i} \varphi(x) \mathrm{d} x \\
= & \int_{\Omega}\left(\mathcal{A}_{i j}^{0} \partial_{j} u^{0}(x)-\mathcal{A}_{i j}^{\varepsilon}(x) \partial_{j} u^{0}(x)-\mathcal{A}_{i j}^{\varepsilon}(x) \varepsilon \partial_{j}\left(\partial_{k} u^{0}(x) w^{k}\left(\frac{x}{\varepsilon}\right)\right)\right) \partial_{i} \varphi(x) \mathrm{d} x \\
& -\int_{\Omega} \mathcal{A}_{i j}^{\varepsilon}(x) \varepsilon \partial_{j}\left(\partial_{k} u^{0}(x) w^{b l, k}\left(\frac{x}{\varepsilon}\right)\right) \partial_{i} \varphi(x) \mathrm{d} x \\
= & \int_{\Omega}\left(\mathcal{A}_{i j}^{0}-\mathcal{A}_{i j}^{\varepsilon}(x)-\mathcal{A}_{i k}^{\varepsilon}(x) \partial_{y_{k}} w^{j}\left(\frac{x}{\varepsilon}\right)\right) \partial_{j} u^{0}(x) \partial_{i} \varphi(x) \mathrm{d} x \\
& -\varepsilon \int_{\Omega} \mathcal{A}_{i j}^{\varepsilon}(x) \partial_{j k} u^{0}(x)\left(w^{k}+w^{b l, k}\right)\left(\frac{x}{\varepsilon}\right) \partial_{i} \varphi(x) \mathrm{d} x \\
& -\int_{\Omega} \mathcal{A}_{i j}^{\varepsilon}(x) \partial_{y_{j}} w^{b l, k}\left(\frac{x}{\varepsilon}\right) \partial_{k} u^{0}(x) \partial_{i} \varphi(x) \mathrm{d} x .
\end{aligned}
$$

For the first term on the right hand side, we use the same technique as in Theorem A.1 from the Appendix in [17], see also [16], in order to obtain:

$$
\int_{\Omega}\left(\mathcal{A}_{i j}^{0}-\mathcal{A}_{i j}^{\varepsilon}(x)-\mathcal{A}_{i k}^{\varepsilon}(x) \partial_{y_{k}} w^{j}\left(\frac{x}{\varepsilon}\right)\right) \partial_{j} u^{0}(x) \partial_{i} \varphi(x) \mathrm{d} x \leq O\left(\varepsilon\left\|\nabla^{2} u^{0}\right\|_{L_{2}(\Omega)}\|\nabla \varphi\|_{L_{2}(\Omega)}\right)+\sigma(R),
$$

with $\sigma(R) \rightarrow 0$ for $R \rightarrow \infty$. For the second term, we observe that by the weak maximum principle the $w^{b l, k}$ are bounded in $L_{\infty}$, and we can estimate this term by $C \varepsilon\left\|\nabla^{2} u^{0}\right\|_{L_{2}(\Omega)}\|\nabla \varphi\|_{L_{2}(\Omega)}$. To estimate the third term in (15), we use the fact that the $w^{b l, k}$ are solutions of the boundary layer-problems (7) and therefore

$$
\partial_{y_{i}}\left(\mathcal{A}_{i j}(y) \partial_{y_{j}} w^{b l, k}(y)\right)=0
$$

The subsequent Lemma 2.6 then yields the existence of skew-symmetric tensors $\left(\beta_{i l}^{k}\right) \in\left(W_{\gamma}(Z)\right)^{n^{2}}$ such that

$$
\partial_{y_{l}} \beta_{i l}^{k}(y)=\mathcal{A}_{i j}(y) \partial_{y_{j}} w^{b l, k}(y)
$$

Taking into account that $\partial_{y_{k}}=\varepsilon \partial_{x_{k}}$, we obtain for the third term in (15):

$$
\begin{aligned}
\int_{\Omega} \mathcal{A}_{i j}\left(\frac{x}{\varepsilon}\right) \partial_{y_{j}} w^{b l, k}\left(\frac{x}{\varepsilon}\right) \partial_{k} u^{0}(x) \partial_{i} \varphi(x) & \\
= & \int_{\Omega \cap B_{R}} \partial_{y_{l}} \beta_{i l}^{k}\left(\frac{x}{\varepsilon}\right) \partial_{k} u^{0}(x) \partial_{i} \varphi(x)+\int_{\Omega \backslash B_{R}} \mathcal{A}_{i j}\left(\frac{x}{\varepsilon}\right) \partial_{y_{j}} w^{b l, k}\left(\frac{x}{\varepsilon}\right) \partial_{k} u^{0}(x) \partial_{i} \varphi(x) \\
= & \varepsilon \int_{\Omega \cap B_{R}} \partial_{l}\left(\beta_{i l}^{k}\left(\frac{x}{\varepsilon}\right) \partial_{k} u^{0}(x)\right) \partial_{i} \varphi(x)-\varepsilon \int_{\Omega \cap B_{R}} \beta_{i p}^{k}\left(\frac{x}{\varepsilon}\right) \partial_{l k} u^{0}(x) \partial_{i} \varphi(x) \\
& +\int_{\Omega \backslash B_{R}} \mathcal{A}_{i j}\left(\frac{x}{\varepsilon}\right) \partial_{y_{j}} w^{b l, k}\left(\frac{x}{\varepsilon}\right) \partial_{k} u^{0}(x) \partial_{i} \varphi(x) .
\end{aligned}
$$


Due to the Hölder-regularity of the coefficients $\mathcal{A}_{i j}$, the $\nabla w^{b l, k}$ and $\beta_{i l}^{k}$ are bounded in $L_{\infty}$. Together with the regularity of $u^{0}$ and $\varphi$, this implies that the second term on the right hand side of the relation above is of the desired form and that the third term tends to zero for $R \rightarrow \infty$. Integrating by parts for $\varphi \in \dot{H}_{0}^{1}(\Omega) \cap\left\{C^{\infty}(\bar{\Omega}) \mid\right.$ $\operatorname{supp}(\varphi) \cap \partial \Omega=\emptyset\}$, we get:

$$
\varepsilon \int_{\Omega \cap B_{R}} \partial_{l}\left(\beta_{i l}^{k}\left(\frac{x}{\varepsilon}\right) \partial_{k} u^{0}(x)\right) \partial_{i} \varphi(x)=\varepsilon \int_{\Omega \cap B_{R}} \beta_{i l}^{k}\left(\frac{x}{\varepsilon}\right) \partial_{k} u^{0}(x) \partial_{l i} \varphi(x)-\varepsilon \int_{\Omega \cap \partial B_{R}} \beta_{i l}^{k}\left(\frac{x}{\varepsilon}\right) \partial_{k} u^{0}(x) \partial_{i} \varphi(x) n_{l} .
$$

Now the first term is zero because of the skew-symmetry of $\beta_{i l}^{k}$, and the second term tends to zero for $R \rightarrow \infty$. Since the subspace

$$
\left\{\varphi \in C^{\infty}(\bar{\Omega}) \mid\|\nabla \varphi\|_{L_{2}(\Omega)} \leq C, \operatorname{supp}(\varphi) \cap \partial \Omega=\emptyset\right\}
$$

is dense in $\dot{H}_{0}^{1}(\Omega)$ with respect to the norm $\|\cdot\|_{\dot{H}^{1}(\Omega)}$, we can put

$$
\varphi(x)=u^{\varepsilon}(x)-u^{0}(x)-\varepsilon \partial_{k} u^{0}(x)\left(w^{k}+w^{b l, k}\right)\left(\frac{x}{\varepsilon}\right)
$$

in (15). Then, for $R \rightarrow \infty$, and by using the ellipticity of the coefficients $\mathcal{A}_{i j}^{\varepsilon}$, we get the desired energyestimate (14).

In the proof of Theorem 2.5 we have used the following lemma. We will also use it in Section 4.2 when we deal with the case of a domain with a curved boundary.

Lemma 2.6. Let $h_{i}, i=1, \ldots, n$ be defined on the boundary layer cell and have the following properties:

1. There exists $\gamma>0$ such that $\left(\mathrm{e}^{\gamma y_{n}} h_{i}\right) \in\left(L_{2}(Z)\right)^{n}$

2. $\partial_{y_{i}} h_{i}=0$ in the sense of distributions.

Then there exists a skew-symmetric matrix $\left(\beta_{i l}\right)$ with $\left(\mathrm{e}^{\gamma y_{n}} \beta_{i l}\right) \in\left(H_{2}^{1}(Z)\right)^{n^{2}}$ such that

$$
\partial_{y_{l}} \beta_{i l}=h_{i}, i=1, \ldots, n .
$$

Proof. Let $\zeta_{i}, i=1, \ldots, n$ be solutions of the following problems:

$\Delta \zeta_{i}=h_{i}$ in Z;

$\zeta_{i}\left(y^{\prime}, 0\right)=0$ for $y^{\prime} \in Z^{\prime}$ and $i=1, \ldots, n-1$;

$\frac{\partial \zeta_{n}}{\partial y_{n}}\left(y^{\prime}, 0\right)=0$ for $y^{\prime} \in Z^{\prime}$;

$\zeta_{i}$ periodic in $y^{\prime}$;

$\nabla \zeta_{i}$ decreases exponentially as $y_{n} \rightarrow \infty$.

For $i=1, \ldots, n-1$, the existence and uniqueness of $\zeta_{i} \in W_{\gamma}(Z)$ follows directly from Theorem 10.1 in [14]. To study the problem for $\zeta_{n}$, we first have to prove that $h_{n}$ satisfies the compatibility condition:

$$
\int_{Z} h_{n}(y) \mathrm{d} y=\int_{\partial Z} n_{i} \frac{\partial \zeta_{n}}{\partial y_{i}}=\int_{Z^{\prime}} \frac{\partial \zeta_{n}}{\partial y_{n}}\left(y^{\prime}, 0\right) \mathrm{d} \sigma=0 .
$$

Using the fact that $\left(h_{i}\right)$ is divergence-free and periodic in $y^{\prime}$, we get:

$$
\int_{Z^{\prime}} \int_{a}^{b} \frac{\partial h_{n}(y)}{\partial y_{n}}=-\sum_{i=1}^{n-1} \int_{Z^{\prime}} \int_{a}^{b} \frac{\partial h_{i}(y)}{\partial y_{i}}=0 .
$$

Therefore

$$
\int_{Z^{\prime}} h_{n}\left(y^{\prime}, a\right)=\int_{Z^{\prime}} h_{n}\left(y^{\prime}, b\right), \quad \forall a, b>0 .
$$


If we now let $b \rightarrow \infty$, we have

and by integrating over $\alpha$ from 0 to $\infty$, we get

$$
\int_{Z^{\prime}} h_{n}\left(y^{\prime}, \alpha\right)=0 \quad \forall \alpha>0,
$$

$$
\int_{Z} h_{n}(y) \mathrm{d} y=\int_{0}^{\infty} \int_{Z^{\prime}} h_{n}\left(y^{\prime}, \alpha\right) \mathrm{d} y^{\prime} \mathrm{d} \alpha=0 .
$$

Existence and uniqueness of $\zeta_{n}$ in the space

$$
\dot{W}_{\gamma}(Z)=\left\{w \in L_{2}(] 0, R\left[, H_{\mathrm{per}}^{1}\left(Z^{\prime}\right)\right) / \mathbb{R}, \forall R<\infty, \mathrm{e}^{\gamma y_{n}} \nabla w \in L_{2}(Z)\right\}
$$

now follow from Tartar's Lemma A.1 by using the techniques of Theorem 10.1 in [14]. By using standard techniques, we can also prove the regularity of the second derivatives of $\vec{\zeta}$, i.e., that $\mathrm{e}^{\gamma y_{n}} \nabla^{2} \zeta_{i} \in\left(L_{2}(Z)\right)^{n^{2}}$.

Let us now define the matrix $\beta_{i l}$ by:

$$
\beta_{i l}:=\partial_{y_{l}} \zeta_{i}-\partial_{y_{i}} \zeta_{l}
$$

Then, obviously, $\beta_{i l}$ is skew-symmetric, and due to the regularity of the $\zeta_{i}$, it satisfies $\left(\mathrm{e}^{\gamma y_{n}} \beta_{i l}\right) \in\left(H_{2}^{1}(Z)\right)^{n^{2}}$. Let us now verify the relation (16):

$$
\partial_{y_{l}} \beta_{i l}=\partial_{y_{l}} \partial_{y_{l}} \zeta_{i}-\partial_{y_{l}} \partial_{y_{i}} \zeta_{l}=h_{i}-\partial_{y_{l}} \partial_{y_{i}} \zeta_{l} .
$$

We have to show that $\partial_{y_{i}} \Xi=0$, for $\Xi:=\sum_{l=1}^{n} \partial_{y_{l}} \zeta_{l}$. Using the properties of the solutions $\zeta_{l}$ and the relation $\partial_{y_{i}} h_{i}=0$, we have $\Xi$ as the unique solution of the following problem:

$$
\begin{aligned}
& \Delta \Xi=0 \text { in } Z \\
& \Xi\left(y^{\prime}, 0\right)=0 \text { for } y^{\prime} \in Z^{\prime} \\
& \Xi \text { is periodic in } y^{\prime} \\
& \nabla \Xi \text { decreases exponentially for } y_{n} \rightarrow \infty
\end{aligned}
$$

and is necessarily equal to zero. Thus, $\left(\beta_{i l}\right)$ satisfies the desired relation.

\section{THE CONSTRUCTION OF BOUNDARY CORRECTORS FOR DOMAINS WITH CURVED BOUNDARY}

The results concerning boundary correctors which were presented in the previous section have been obtained for a domain with a very special geometry. Now it is of interest whether the techniques developed there can also be used for more general domains. Obviously, if we drop the special assumptions on the geometry of the domain, we lose the periodicity properties parallel to the boundary, and the boundary layer cell becomes a half space. It is therefore clear that the techniques of the previous section can be applied to arbitrary domains only if we generalize the boundary layers such that the case of an infinite period is included.

In this section, we will construct boundary correctors for domains $\Omega$ of class $C^{2}$ defined in the following.

Definition 3.1. We say that a domain $\Omega \subset \mathbb{R}^{n}$ is of class $C^{k}$, for $k \in \mathbb{N}, k \geq 1$, if for any $\omega \in \partial \Omega$ there exist a neighborhood $U_{\omega}$ and a orthogonal coordinate transform $A_{\omega}: \mathbb{R}^{n} \rightarrow \mathbb{R}^{n}$, two numbers $\alpha_{\omega}, \beta_{\omega}>0$ and a function $a_{\omega}: \mathbb{R}^{n-1} \rightarrow \mathbb{R}$, such that, if $z_{1}, \ldots, z_{n}$ are the new Cartesian coordinates given by the transformation $A_{\omega}$ and $W^{n-1}\left(\alpha_{\omega}\right)$ is the cube:

$$
\left\{\left(z_{1}, \ldots, z_{n-1}\right)|| z_{i} \mid<\alpha_{\omega}, i=1, \ldots, n-1\right\} \text { in } \mathbb{R}^{n-1},
$$




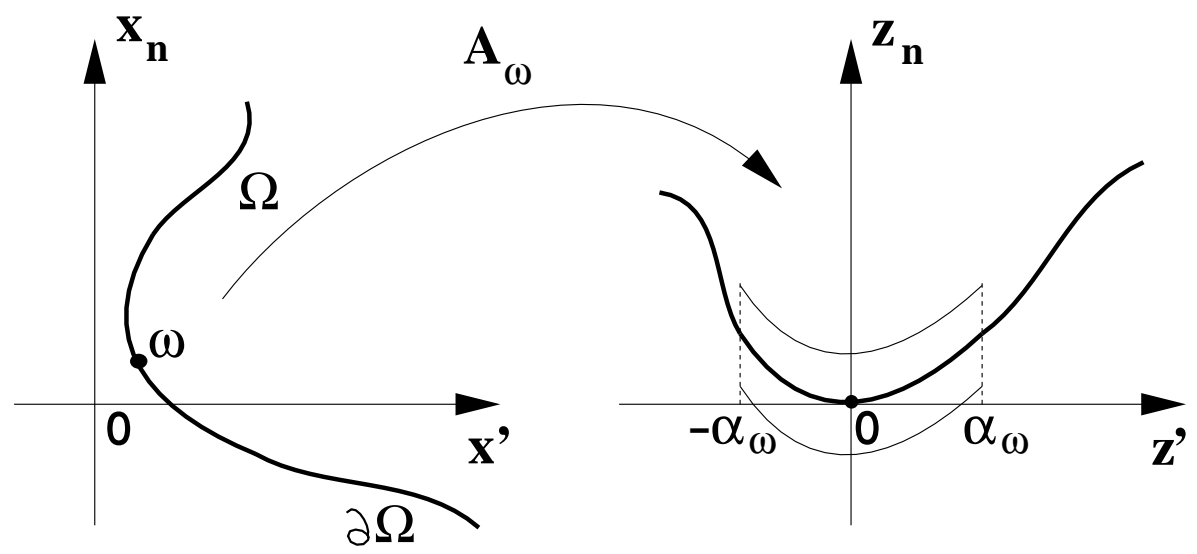

Figure 2. Domain of class $C^{k}$.

then the following properties hold:

1. $a_{\omega} \in C^{k}\left(W^{n-1}\left(\alpha_{\omega}\right)\right)$ with $K_{\omega}:=\left\|a_{\omega}\right\|_{C^{k}\left(W^{n-1}\left(\alpha_{\omega}\right)\right)}<\infty, a_{\omega}(0)=0$, and $\nabla a_{\omega}(0)=0$.

2. $A_{\omega}(\omega)=0$

3. The boundary portion $A_{\omega}\left(U_{\omega} \cap \partial \Omega\right)$ is given by the function $y_{n}=a_{\omega}\left(z_{1}, \ldots, z_{n-1}\right)$, i.e.,

$$
A\left(U_{\omega} \cap \partial \Omega\right)=\left\{\left(z_{1}, \ldots, z_{n}\right) \mid\left(z_{1}, \ldots, z_{n-1}\right) \in W^{n-1}\left(\alpha_{\omega}\right), y_{n}=a_{\omega}\left(z_{1}, \ldots, z_{n-1}\right)\right\}
$$

4. The intersection $A_{\omega}\left(U_{\omega} \cap \Omega\right)$ is given by:

$$
A_{\omega}\left(U_{\omega} \cap \Omega\right)=\left\{\left(z_{1}, \ldots, z_{n}\right) \mid\left(z_{1}, \ldots, z_{n-1}\right) \in W^{n-1}\left(\alpha_{\omega}\right), 0<z_{n}-a_{\omega}\left(z_{1}, \ldots, z_{n-1}\right)<\beta_{\omega}\right\}
$$

5. The intersection $A_{\omega}\left(U_{\omega} \cap \boldsymbol{C} \Omega\right)$ can be described by:

$$
A_{\omega}\left(U_{\omega} \cap \boldsymbol{C} \Omega\right)=\left\{\left(z_{1}, \ldots, z_{n}\right) \mid\left(z_{1}, \ldots, z_{n-1}\right) \in W^{n-1}\left(\alpha_{\omega}\right),-\beta_{\omega}<z_{n}-a_{\omega}\left(z_{1}, \ldots, z_{n-1}\right)<0\right\} .
$$

We say that $\Omega$ is uniformly of the class $C^{2}$ if we can choose $\alpha_{\omega}=\alpha, \beta_{\omega}=\beta$ and $K_{\omega}=K$ for $\alpha, \beta, K>0$.

Remark 3.2. The definition above is equivalent to the usual definition for domains of class $C^{k}$, see [22].

Theorem 3.3 (Tubular neighborhoods). Let $\Omega \subset \mathbb{R}^{n}$ be a domain uniformly of class $C^{k}, k \geq 2$ and $\vec{v} \in$ $C^{2}(\partial \Omega)$ be a vector-field such that for each $\omega \in \partial \Omega$ we have $|\vec{v}(\omega)|=1$ and

$$
\left(A_{\omega} \vec{v}\right)_{n}(0) \geq \rho>0
$$

where the transformation $A_{\omega}$ is the same as in Definition 3.1. Then there exist a number $\delta>0$, $\delta=$ $\delta\left(\alpha, \beta, K,\|\vec{v}\|_{C^{2}(\partial \Omega}, \rho\right)$, and a neighborhood $V_{\delta}$ of $\partial \Omega$ such that the transformation

$$
\Phi: \partial \Omega \times]-\delta, \delta\left[\longrightarrow V_{\delta},(\omega, t) \mapsto \omega+t \vec{v}(\omega)\right.
$$

is a $C^{2}$-diffeomorphism.

Proof. The assertion of the theorem will be proven if for each $\omega \in \partial \Omega$ we can find a neighborhood $W(\omega) \times]-\delta, \delta[$ of the point $(\omega, 0) \in \partial \Omega \times \mathbb{R}$ with $\delta=\delta(\alpha, \beta, K, \rho)$ such that:

(a) the transformation

$$
\Phi: W(\omega) \times]-\delta, \delta[\longrightarrow \Phi(W(\omega) \times]-\delta, \delta[)
$$

is a $C^{2}$-diffeomorphism, and 
(b) on the union $\left.\bigcup_{\omega \in \partial \Omega} W(\omega) \times\right]-\delta, \delta\left[\right.$ the transformation $\Phi$ remains bijective, and is thus a $C^{2}$-diffeomorphism from $\partial \Omega \times]-\delta, \delta[$ into $\Phi(\partial \Omega \times]-\delta, \delta[)$.

Because the domain $\Omega$ is uniformly of class $C^{2}$ in the sense of Definition 3.1, it is sufficient to show the assertion (a) in a coordinate-neighborhood

$$
U_{\alpha \beta}(0)=\left\{\left(z^{\prime}, z_{n}\right):\left|z^{\prime}\right|<\alpha,\left|z_{n}-a_{\omega}\left(z^{\prime}\right)\right|<\beta\right\} .
$$

In this coordinate-neighborhood, the function $\Phi$ is given by:

$$
\left(\begin{array}{l}
z_{1} \\
\cdots \\
z_{n-1} \\
t
\end{array}\right) \mapsto\left(\begin{array}{l}
z_{1} \\
\cdots \\
z_{n-1} \\
a_{\omega}\left(z^{\prime}\right)
\end{array}\right)+t\left(\begin{array}{l}
\left(A_{\omega} \vec{v}\right)_{1}\left(z^{\prime}, a_{\omega}\left(z^{\prime}\right)\right) \\
\cdots \\
\left(A_{\omega} \vec{v}\right)_{n-1}\left(z^{\prime}, a_{\omega}\left(z^{\prime}\right)\right) \\
\left(A_{\omega} \vec{v}\right)_{n}\left(z^{\prime}, a_{\omega}\left(z^{\prime}\right)\right)
\end{array}\right) .
$$

Now it is possible to choose a smaller neighborhood $]-\alpha^{\prime}, \alpha^{\prime}\left[{ }^{n-1} \times\right]-\beta^{\prime}, \beta^{\prime}\left[\subset U_{\alpha \beta}(0)\right.$, with $\alpha^{\prime}$ and $\beta^{\prime}$ depending on $\alpha, \beta$ and $\mathrm{K}$, such that $\left.\Phi\right|_{]-\alpha^{\prime}, \alpha^{\prime}\left[{ }^{n-1} \times\right]-\beta^{\prime}, \beta^{\prime}[}$ takes values in $U_{\alpha \beta}(0)$.

Due to the smoothness assumptions on the function $a_{\omega}$ and on the vector-field $\vec{v}$, it follows that $\Phi \in C^{2}(]-\alpha^{\prime}, \alpha^{\prime}\left[{ }^{n-1} \times\right]-\beta^{\prime}, \beta^{\prime}\left[, U_{\alpha \beta}(0)\right)$, and there exists a constant $C_{\Phi}=C_{\Phi}\left(K,\|\vec{v}\|_{C^{2}(\partial \Omega)}\right)$ with

$$
\|\Phi\|_{C^{2}(]-\alpha^{\prime}, \alpha^{\prime}[n-1 \times]-\beta^{\prime}, \beta^{\prime}[)} \leq C_{\Phi} .
$$

In order to show that $\Phi$ is a $C^{2}$-diffeomorphism, we intend to use the Inverse Mapping Theorem. Therefore let us first calculate the Jacobian of $\Phi$ in 0 :

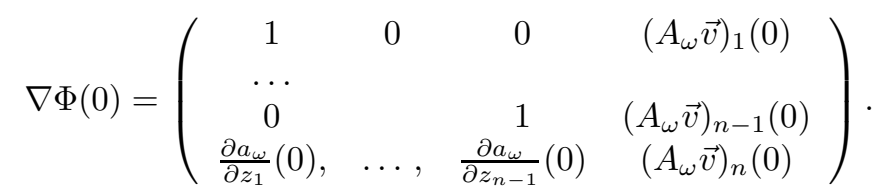

Due to the relation (17) and the properties of $a_{\omega}$ from Definition 3.1, we have that

$$
\operatorname{det} \nabla \Phi(0)=\left(A_{\omega} \vec{v}\right)_{n}(0) \geq \rho>0 .
$$

One also easily sees that

$$
\left\|\nabla \Phi(0)^{-1}\right\| \leq \frac{\sqrt{2}}{\rho}
$$

Then, by the Inverse Mapping Theorem A.5, there exists

$$
r=r\left(C_{\Phi}, \rho, \alpha^{\prime}, \beta^{\prime}\right)
$$

with $]-r, r\left[{ }^{n} \subset\right]-\alpha^{\prime}, \alpha^{\prime}\left[{ }^{n-1} \times\right]-\beta^{\prime}, \beta^{\prime}[$ such that

$$
\left.\left.\Phi\right|_{]-r, r[n}:\right]-r, r\left[{ }^{n} \rightarrow \Phi(]-r, r\left[^{n}\right) \subset U_{\alpha, \beta}(0)\right.
$$

is a $C^{2}$-diffeomorphism.

If we set

$$
\delta=\frac{r}{2},
$$

the assertion of (b) is also satisfied, and the proof is complete. 


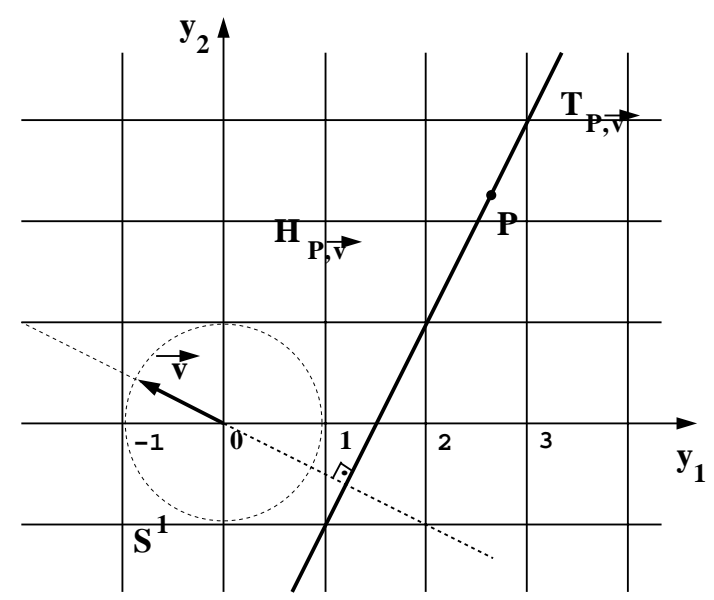

Figure 3. The domain $H_{P, \vec{v}}$.

Remark 3.4. For domains of class $C^{k}, k \geq 3$, a possible choice for the vector-field $\vec{v}$ would be the inner normal $\vec{\nu}$ to $\partial \Omega$, locally given by:

$$
\vec{\nu}=\left(\nu_{1}, \ldots, \nu_{n}\right)=\frac{1}{K}\left(-\frac{\partial a_{\omega}}{\partial z_{1}}, \ldots,-\frac{\partial a_{\omega}}{\partial z_{n-1}}, 1\right), \text { where } K=\left[1+\sum_{i=1}^{n-1}\left(\frac{\partial a_{\omega}}{\partial z_{i}}\right)^{2}\right]^{\frac{1}{2}}
$$

After these considerations on the domain, let us turn our attention to the construction of the boundary correctors. As we have already mentioned in the beginning of this section, in order to define boundary correctors for general smooth domains, we first have to extend the notion of boundary layer. This will be done by help of the following construction (see Fig. 3). For any point $P \in \mathbb{R}^{n}$ and vector $\vec{v} \in S^{n-1}$, we denote by $T_{P, \vec{v}}$ the hyperplane containing the point $\mathrm{P}$ and having the normal vector $\vec{v}$ :

$$
T_{P, \vec{v}}=\left\{y \in \mathbb{R}^{n} \mid(y-P, v)=0\right\}
$$

and by $H_{P, \vec{v}}$ be the half space with boundary $T_{P, \vec{v}}$ and having $\vec{v}$ as inner normal:

$$
H_{P, \vec{v}}=\left\{y \in \mathbb{R}^{n} \mid(y-P, v)>0\right\} .
$$

Let the coefficients $\mathcal{A}_{i j}(y)$ be defined by (i), (ii), (iii), and $w^{k} \in H_{\mathrm{per}}^{1}(Y)$ be the solutions of the cellproblems $\left(C^{k}\right)$.

Definition 3.5. We define the functions $w_{k}^{P, \vec{v}}: H_{P, \vec{v}} \rightarrow \mathbb{R}$ to be the solutions of the following problems:

$$
\begin{array}{ll}
\partial_{y_{i}}\left(\mathcal{A}_{i j}(y) \partial_{y_{j}} w_{k}^{P, \vec{v}}\right)=0, & y \in H_{P, \vec{v}} \\
w_{k}^{P, \vec{v}}(y)=-w^{k}(y), & y \in T_{P, \vec{v}} \\
\left\|w_{k}^{P, \vec{v}}\right\|_{L_{\infty}\left(H_{P, \vec{v}}\right)} \leq C . &
\end{array}
$$


Since the restriction of the cell-solution $w^{k}$ to the boundary $T_{P, \vec{v}}$ is not periodic in general, we cannot use Theorem 2.2 to obtain existence and uniqueness of the solutions $w_{k}^{P, \vec{v}}$ to problem (19). Instead, we use the theory of de Giorgi, Nash and Moser to obtain the following theorem:

Theorem 3.6. Let the coefficients $\mathcal{A}_{i j} \in C^{0, \kappa}\left(\mathbb{R}^{n}\right)$. Then there exists a unique solution $w_{k}^{P, \vec{v}} \in C^{1, \kappa}\left(\bar{H}_{P, \vec{v}}\right)$.

Proof. The existence of the solutions $w_{k}^{P, \vec{v}}$ can be proven by using Perron's method of subsolutions (resp. supersolutions), see for example [11]. The uniqueness of the solution $w_{k}^{P, \vec{v}}$ follows from Theorem A.4 in the following way: Let $w^{1}$ and $w^{2}$ be two solutions for the problem (19). Then the difference $w^{1,2}=w^{1}-w^{2}$ satisfies

$$
\begin{array}{ll}
\partial_{y_{i}}\left(\mathcal{A}_{i j}(y) \partial_{y_{j}} w^{1,2}\right)=0, & y \in H_{P, \vec{v}} \\
w^{1,2}(y)=0, & y \in T_{P, \vec{v}} \\
\left\|w^{1,2}\right\|_{L_{\infty}\left(H_{P, \vec{v}}\right)} \leq C . &
\end{array}
$$

Consider now $y \in H_{P, \vec{v}}$ and let $y_{0} \in T_{P, \vec{v}}$ be the orthogonal projection of $y$ on $T_{P, \vec{v}}$. Let $R>0$ be such that $y \in$ $B_{R}\left(y_{0}\right)$. For each $R_{0}>R, w^{1,2}$ lies in $H^{1,2}\left(H_{P, \vec{v}} \cap B_{2 R_{0}}\left(y_{0}\right)\right)$, and it vanishes on $\partial\left(H_{P, \vec{v}} \cap B_{2 R_{0}}\left(y_{0}\right)\right) \cap B_{\sqrt{R R_{0}}}\left(y_{0}\right)$. Then by Theorem A.4, for each $y^{\prime} \in H_{P, \vec{v}} \cap B_{R}\left(y_{0}\right)$ we have that

$$
\left|w^{1,2}(y)-w^{1,2}\left(y^{\prime}\right)\right| \leq C\left[\frac{R}{R_{0}}\right]^{\kappa} \sup _{H_{P, \vec{v}} \cap B_{R_{0}}\left(y_{0}\right)}\left|w^{1,2}\right| .
$$

For $R_{0} \rightarrow \infty$, the right hand side of the relation above tends to zero. Since $w^{1,2}=0$ on $T_{P, \vec{v}}$, we obtain $w^{1,2} \equiv 0$.

By help of the functions $w_{k}^{P, \vec{v}}$, we can give the following generalization for the boundary layers: Let $\Omega$ be a domain of class $C^{2}$. In each $\omega \in \partial \Omega$, we consider the inner unit normal $\vec{\nu}(\omega)$.

Definition 3.7. We define the generalized boundary layers in a point $\omega \in \partial \Omega$ as the unique solutions

$$
w_{k}^{\frac{\omega}{\varepsilon}, \vec{\nu}(\omega)}: H_{\frac{\omega}{\varepsilon}, \vec{\nu}(\omega)} \rightarrow \mathbb{R}
$$

of the problems (19), corresponding to the parameters $\left(\frac{\omega}{\varepsilon}, \vec{\nu}(\omega)\right)$.

Remark 3.8. For the special case of Section 2, the generalized boundary layers coincide with the classical, periodic boundary layers $w^{k, b l}$ defined on the standard boundary layer-cell $\left.Z=\right] 0,1\left[^{(n-1)} \times\right] 0, \infty[$ and extended periodically to the half space.

Using the generalized boundary layers, we define the boundary corrector $w^{\varepsilon}$ by the formula:

$$
w^{\varepsilon}(x)= \begin{cases}\varepsilon \partial_{k} u^{0}(x) w_{k}^{\frac{\omega}{\varepsilon}, \vec{\nu}(\omega)}\left(\frac{x}{\varepsilon}\right) \eta(t) & x=\Phi(\omega, t) \in \Omega_{\delta} \\ 0 & x \in \Omega \backslash \Omega_{\delta}\end{cases}
$$

where the subdomain $\Omega_{\delta}$ is the intersection of the tubular neighborhood $V_{\delta}$ from Theorem 3.3 with $\Omega$ and is given by:

$$
\Omega_{\delta}:=\{x \in \Omega \mid x=\Phi(\omega, t), \omega \in \partial \Omega, 0<t<\delta\},
$$

and $\eta$ is a cut-off function defined by:

$$
\eta \in C^{\infty}(\Omega,[0,1]), \quad \eta(t)=\left\{\begin{array}{ll}
1 & 0<t<\frac{\delta}{2} \\
0 & \delta<t
\end{array} .\right.
$$


If we compare the boundary correctors given by (11), respectively (21), we see that in the case of curved boundaries they have a more complicated form. In (11) they are determined by the values of the boundary layers on a standard boundary layer cell. For curved boundaries, the boundary corrector is given in each point $x=\Phi(\omega, t)$ by the values of the generalized boundary layers corresponding to the parameters $\frac{\omega}{\varepsilon}, \vec{\nu}(\omega)$.

For the study of the regularity of the boundary corrector with respect to the boundary parameters, we need some knowledge about the decay of the generalized boundary layers $w_{k}^{\frac{\omega}{\varepsilon}, \vec{\nu}(\omega)}$ in the direction normal to the boundary. However, in [17] we have proved that the exponential decay property of the classical boundary layers does not hold in the case of a general domain. Since, in general, we do not have precise decay estimates of the generalized boundary layers, we will restrict to the special case of a layered medium.

\section{THE CASE OF A LAYERED MEDIUM}

In this section, we will study the regularity of the boundary corrector for one class of domains with curved boundaries in the case of a layered medium. Then, we will show that this boundary corrector leads to an error estimate of order $\varepsilon$ with respect to the energy norm. For simplicity, we consider the two-dimensional case here, but all arguments can easily be extended to the multidimensional case.

Let the domain $\Omega \subset \mathbb{R}^{2}$ (see Fig. 4) be given by

$$
\Omega=\left\{x=\left(x_{1}, x_{2}\right) \mid x_{1} \in \mathbb{R}, x_{2}>g\left(x_{1}\right)\right\},
$$

where $g \in C^{2}(\mathbb{R})$ satisfies

$$
\|g\|_{C^{2}(\mathbb{R})} \leq G<\infty
$$

and set

$$
\phi_{g}:=\arctan \|\nabla g\|_{C^{0}(\mathbb{R})} .
$$

We start from the microscopic problem:

For $f \in C_{0}^{\infty}(\bar{\Omega})$, find $u^{\varepsilon} \in \dot{H}_{0}^{1}(\Omega)$, such that

$$
\int_{\Omega} \mathcal{A}_{i j}^{\varepsilon}\left(x_{1}\right) \partial_{j} u^{\varepsilon}(x) \partial_{i} \varphi(x) \mathrm{d} x=\int_{\Omega} f(x) \varphi(x) \mathrm{d} x, \forall \varphi \in \dot{H}_{0}^{1}(\Omega)
$$

on the domain $\Omega$ defined in (22). The coefficients $\mathcal{A}_{i j}^{\varepsilon}\left(x_{1}\right)$ describing the layered medium structure satisfy the conditions (i), (ii), (iii) from Section 1.

Since $\Omega$ is smooth, we can construct the boundary corrector analogously to Section 3 . We consider again the solutions $w_{k}^{P, \vec{v}}$ to the auxiliary problems (19). If $\phi$ is the angle between $T_{P, \vec{v}}$ and the $y_{1}$-axes, then the vector $\vec{v}$ is given by $\vec{v}(\phi)=(-\sin \phi, \cos \phi)$. Therefore, we will use $P, \phi$ as the parameters determining the solution $w_{k}^{P, \vec{v}}$, which from now on will be denoted by $w_{k}^{P, \phi}$. We will also use the following notations:

$$
H_{P, \phi}:=H_{P, \vec{\nu}(\phi)} \text { and } T_{P, \phi}:=T_{P, \vec{\nu}(\phi)} .
$$

For the special case of a layered medium, we can give the following theorem concerning the behavior at infinity of the solutions $w_{k}^{P, \phi}$.

Theorem 4.1. Let $Z_{\phi}:=Z \cap H_{P, \phi}$ and $|\phi|<\frac{\pi}{2}$. Then for

$$
0<\gamma \leq \gamma_{\phi}:=\frac{\lambda \sqrt{\rho_{1}}}{2 \Lambda}\left(1+\tan ^{2} \phi\right)^{-2}
$$




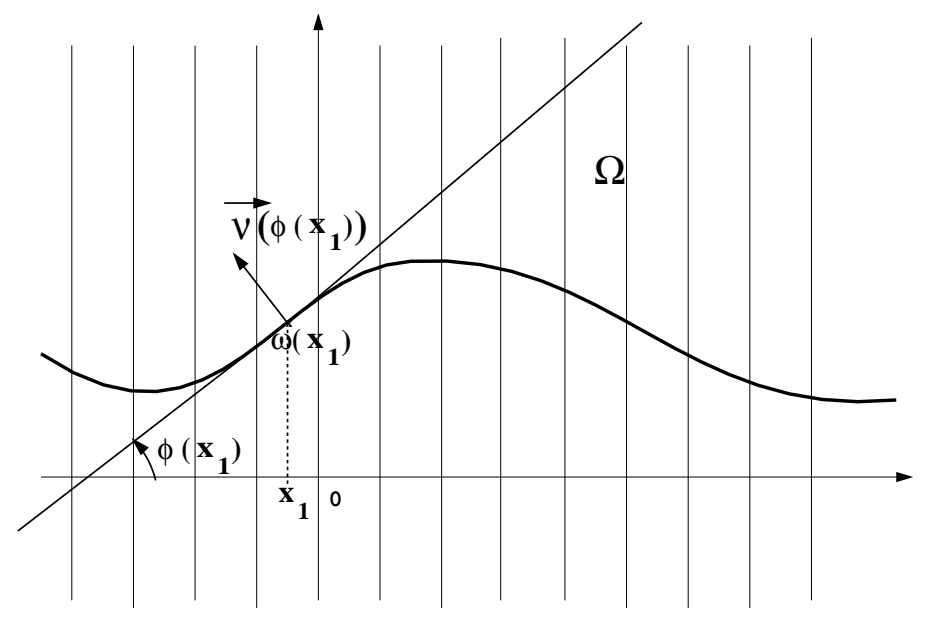

Figure 4. The domain $\Omega$.

the functions $w_{k}^{P, \phi}$ satisfy the following problems:

$$
\begin{aligned}
& \partial_{y_{i}}\left(\mathcal{A}_{i j}\left(y_{1}\right) \partial_{y_{j}} w_{k}^{P, \phi}(y)\right)=0, y \in H_{P, \phi} \\
& w_{k}^{P, \phi}(y)=-w^{k}(y), y \in T_{P, \phi} \\
& w_{k}^{P, \phi} \text { is periodic parallel to } T_{P, \phi} \text { with period } \frac{1}{\cos \phi} \\
& \mathrm{e}^{\gamma y_{2}} \nabla w_{k}^{P, \phi} \in L_{2}\left(Z_{\phi}\right) .
\end{aligned}
$$

Here $\rho_{1}=4 \pi^{2}$ is the smallest nonzero eigenvalue of the Laplace-operator on $[0,1]$ for periodic boundary conditions.

Proof. We intend to apply Theorem 2.2. Therefore, we first transform the problem (26) into a problem on the standard half plane $\mathbb{R} \times(0, \infty)$. For each $P \in \mathbb{R}^{2}$ and $\left.\phi \in\right]-\frac{\pi}{2}, \frac{\pi}{2}[$ we consider the transformation:

$$
\begin{aligned}
\mathbb{R} \times(0, \infty) & \rightarrow H_{P, \phi} \\
\left(\begin{array}{l}
\tilde{y}_{1} \\
\tilde{y}_{2}
\end{array}\right) & \mapsto\left(\begin{array}{l}
y_{1} \\
y_{2}
\end{array}\right)=\left(\begin{array}{l}
\tilde{y}_{1}+P_{1} \\
\tilde{y}_{2}+\tilde{y}_{1} \tan \phi+P_{2}
\end{array}\right) .
\end{aligned}
$$

Under this transformation, the coefficient matrix becomes:

$$
\tilde{\mathcal{A}}^{P, \phi}\left(\tilde{y}_{1}\right)=T^{t}(\phi) \mathcal{A}\left(y_{1}\left(\tilde{y}_{1}\right)\right) T(\phi)
$$

where

$$
T(\phi)=\left(\begin{array}{ll}
\frac{\partial y_{1}}{\partial \ddot{y}_{1}} & \frac{\partial y_{1}}{\partial \ddot{y}_{2}} \\
\frac{\partial y_{2}}{\partial \ddot{y}_{1}} & \frac{\partial y_{2}}{\partial \tilde{y}_{2}}
\end{array}\right)^{-t}=\left(\begin{array}{cc}
1 & -\tan \phi \\
0 & 1
\end{array}\right)
$$

and the functions

$$
\tilde{w}_{k}^{P, \phi}\left(\tilde{y}_{1}, \tilde{y}_{2}\right)=w_{k}^{P, \phi}\left(y_{1}\left(\tilde{y}_{1}, \tilde{y}_{2}\right), y_{2}\left(\tilde{y}_{1}, \tilde{y}_{2}\right)\right)
$$


are the solutions to the following problems:

$$
\begin{aligned}
& \left.\partial_{\tilde{y}_{i}}\left(\tilde{\mathcal{A}}_{i j}^{P, \phi}\left(\tilde{y}_{1}\right) \partial_{\tilde{y}_{j}} \tilde{w}_{k}^{P, \phi}(\tilde{y})\right)=0, \quad \tilde{y} \in \mathbb{R} \times\right] 0, \infty[ \\
& \tilde{w}_{k}^{P, \phi}\left(\tilde{y}_{1}, 0\right)=-\tilde{w}^{k}\left(\tilde{y}_{1}\right) \\
& \tilde{w}_{k}^{P, \phi} \text { periodic in } \tilde{y}_{1} \text { with the period } 1 \\
& \mathrm{e}^{\gamma \tilde{y}_{2}} \nabla \tilde{w}_{k}^{P, \phi} \in L_{2}(\tilde{Z})
\end{aligned}
$$

where $\tilde{Z}=]-\frac{1}{2}, \frac{1}{2}[\times] 0, \infty[$. By Theorem 2.2, for all $\gamma$ satisfying

$$
0<\gamma<\frac{\lambda_{\phi} \sqrt{\rho_{1}}}{2 \Lambda_{\phi}}
$$

the problem (29) has a unique solution in the $W_{\gamma}(\tilde{Z})$, which can be extended by periodicity to the entire domain $\mathbb{R} \times] 0, \infty\left[\right.$. The spectral condition of the matrix $\tilde{\mathcal{A}}^{P, \phi}$ can be estimated by

$$
\frac{\Lambda_{\phi}}{\lambda_{\phi}} \leq \frac{\Lambda}{\lambda}\left(1+\tan ^{2} \phi\right)^{2}
$$

and the assertion of the theorem is proven.

Remark 4.2. From the relation (25) we observe that for $\phi \rightarrow \frac{\pi}{2}$ we have that $\gamma_{\phi} \rightarrow 0$. In [17] we have proven that this result is optimal.

Let us now define the generalized boundary layers and the boundary corrector. We consider in each point $\omega\left(x_{1}\right)=\left(x_{1}, g\left(x_{1}\right)\right) \in \partial \Omega$ the inner unit normal $\vec{\nu}\left(x_{1}\right)=\left(-\sin \phi\left(x_{1}\right), \cos \phi\left(x_{1}\right)\right)$, where $\phi\left(x_{1}\right)$ is the angle between the tangent in $\omega\left(x_{1}\right)$ to $\partial \Omega$ and the $x_{1}$-axis.

Definition 4.3. The generalized boundary layers $w_{k}^{\frac{\omega\left(x_{1}\right)}{\varepsilon}, \phi\left(x_{1}\right)}$ are defined as the solutions to the problems (26) corresponding to the parameters $\frac{\omega\left(x_{1}\right)}{\varepsilon}, \phi\left(x_{1}\right)$.

Remark 4.4. The regularity assumption (23) on the domain $\Omega$ together with Theorem 4.1 imply a uniform exponential decay for the generalized boundary layers $w_{k}^{\frac{\omega\left(x_{1}\right)}{\varepsilon}, \phi\left(x_{1}\right)}$.

For the definition of the boundary corrector, we will use the special geometry of our problem, i.e. the fact that the boundary $\partial \Omega$ is a graph with bounded slope, as well as the layered media structure.

Definition 4.5. The boundary corrector $w^{\varepsilon}: \Omega \rightarrow \mathbb{R}$ is defined as follows:

$$
w^{\varepsilon}(x)=\varepsilon \partial_{k} u^{0}(x) w_{k}^{\frac{\omega\left(x_{1}\right)}{\varepsilon}, \phi\left(x_{1}\right)}\left(\frac{x}{\varepsilon}\right) .
$$

\subsection{Regularity of the boundary corrector}

By help of the boundary corrector $w^{\varepsilon}$, we can give the following approximation for the solution $u^{\varepsilon}$ :

$$
u_{\varepsilon}^{a p p}(x):=u^{0}(x)+\varepsilon \partial_{k} u^{0}(x) w^{k}\left(\frac{x}{\varepsilon}\right)+w^{\varepsilon}(x) .
$$

To determine the accuracy of this approximation with respect to the energy norm, we have to prove an energy estimate for the difference $u^{\varepsilon}-u_{\varepsilon}^{\text {app }}$. The first step toward such an estimate consists in studying the regularity of the boundary corrector $w^{\varepsilon}$. Since the regularity of the homogenized solution $u^{0}$ is well known, we have to 


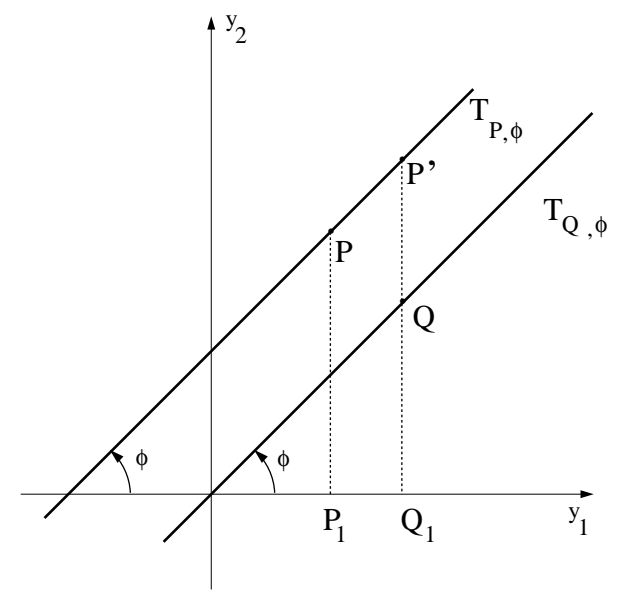

FiguRe 5. Variation of the point $P$.

analyze the regularity of the generalized boundary layers $w_{k}^{\frac{\omega\left(x_{1}\right)}{\varepsilon}, \phi\left(x_{1}\right)}\left(\frac{x}{\varepsilon}\right)$ with respect to $x$. Thus, we first study the differentiability of the auxiliary functions $w_{k}^{P, \phi}$ in the variables $(P, \phi, y)$.

The regularity with respect to $y$ follows from Theorem 2.3 and Theorem 2.4.

Theorem 4.6. If we assume the coefficients $\mathcal{A}_{i j} \in C^{0, \kappa}(\mathbb{R})$ with $0<\kappa<1$, then for each $P \in \mathbb{R}^{n}$ and $\phi \in]-\phi_{g}, \phi_{g}\left[\right.$ the $C^{0, \kappa}$-norm of the gradient of the functions $w_{k}^{P, \phi}$ can be estimated by:

$$
\left\|\nabla w_{k}^{P, \phi}\right\|_{C^{0, \kappa}\left(Z_{\phi}\right)} \leq C\left(\left\|w_{k}^{P, \phi}\right\|_{W_{\gamma}\left(Z_{\phi}\right)}+\left\|w^{k}\right\|_{C^{1, \kappa}(\bar{Y})}\right) .
$$

This result especially implies that the $w_{k}^{P, \phi}$ are Lipschitz-continuous with respect to $y$, with an uniform Lipschitz constant on $Z_{\phi}$.

For the study of the differentiability with respect to $P$ and $\phi$, we will use the following two lemmata.

Lemma 4.7. Let $\mathcal{A}_{i j} \in C^{0, \kappa}(\mathbb{R})$ with $\kappa \in(0,1), P, Q$ be two neighboring points in $\mathbb{R}^{2}$ and let $\left.\phi \in\right]-\phi_{g}, \phi_{g}[$. Then for each $y \in H_{P, \phi} \cap H_{Q, \phi}$ the following estimate holds:

$$
\left|w_{k}^{P, \phi}(y)-w_{k}^{Q, \phi}(y)\right| \leq C\|P-Q\|
$$

Proof. We write the difference above in the following way:

$$
w_{k}^{P, \phi}(y)-w_{k}^{Q, \phi}(y)=w_{k}^{P, \phi}(y)-w_{k}^{P^{\prime}, \phi}(y)+w_{k}^{P^{\prime}, \phi}(y)-w_{k}^{Q, \phi}(y)
$$

where the point $P^{\prime}$ lies on the line $T_{P, \phi}$ and has the first coordinate $Q_{1}$, see Figure 5 . Now we observe that the first difference is zero because $w_{k}^{P, \phi}$ and $w_{k}^{P^{\prime}, \phi}$ are the solutions of the same problem defined on the half space $H_{P, \phi}$. For the second difference, we use the fact that the coefficients and the boundary condition of the problems (26) do not change if we shift the domain $H_{P^{\prime}, \phi}$ by a vector proportional to $\vec{e}_{2}$. Therefore we have that:

$$
w_{k}^{P^{\prime}, \phi}(y)-w_{k}^{Q, \phi}(y)=w_{k}^{P^{\prime}, \phi}(y)-w_{k}^{P^{\prime}, \phi}\left(y+\left(P^{\prime}-Q\right)\right)
$$




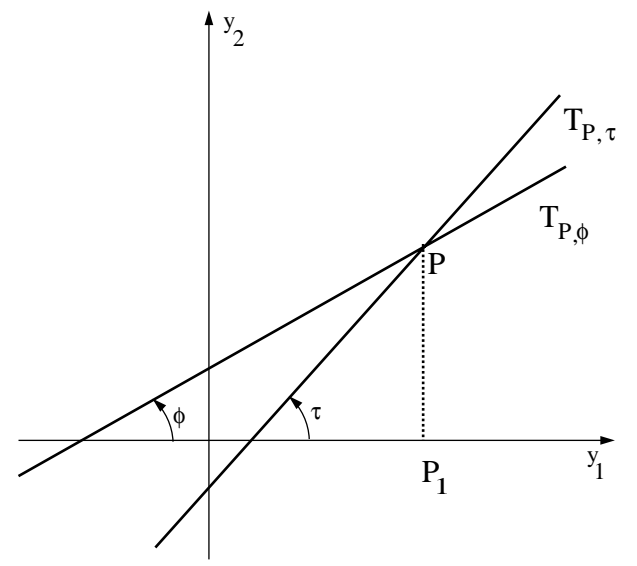

Figure 6. Variation of the angle $\phi$.

and using the Lipschitz-continuity of $w_{k}^{P^{\prime}, \phi}$, we obtain:

$$
\begin{aligned}
\left|w_{k}^{P^{\prime}, \phi}(y)-w^{Q, \phi}(y)\right| & \leq C\left|P_{2}^{\prime}-Q_{2}\right| \leq C\left|P_{2}^{\prime}-P_{2}\right|+\left|P_{2}-Q_{2}\right| \\
& \leq C|\tan \phi|\left|P_{1}-Q_{1}\right|+C\left|P_{2}-Q_{2}\right| \\
& \leq C\|P-Q\| .
\end{aligned}
$$

Lemma 4.8. Let $\mathcal{A}_{i j} \in C^{0, \kappa}(\mathbb{R})$ with $\kappa \in(0,1), P \in \mathbb{R}^{2}$ and $\phi, \tau$ be two neighboring angles in $]-\phi_{g}$, $\phi_{g}[$, see Figure 6. Then for each $y \in H_{P, \phi} \cap H_{P, \tau}$ the following estimate holds:

$$
\left|w_{k}^{P, \phi}(y)-w_{k}^{P, \tau}(y)\right| \leq C\left|y_{1}-P_{1}\right||\phi-\tau|+C|\phi-\tau| .
$$

Proof. Again let us use the coordinates $\left(\tilde{y}_{1}, \tilde{y}_{2}\right)$ from (27), and write the difference above in the following way:

$$
\begin{aligned}
w_{k}^{P, \phi}(y)-w_{k}^{P, \tau}(y)= & \tilde{w}_{k}^{P, \phi}\left(y_{1}-P_{1}, y_{2}+\left(y_{1}-P_{1}\right) \tan \phi-P_{2}\right) \\
& -\tilde{w}_{k}^{P, \tau}\left(y_{1}-P_{1}, y_{2}+\left(y_{1}-P_{1}\right) \tan \tau-P_{2}\right) .
\end{aligned}
$$

Now we split this difference and obtain:

$$
\begin{aligned}
w_{k}^{P, \phi}(y)-w_{k}^{P, \tau}(y)= & \tilde{w}_{k}^{P, \phi}\left(y_{1}-P_{1}, y_{2}+\left(y_{1}-P_{1}\right) \tan \phi-P_{2}\right) \\
& -\tilde{w}_{k}^{P, \phi}\left(y_{1}-P_{1}, y_{2}+\left(y_{1}-P_{1}\right) \tan \tau-P_{2}\right) \\
& +\tilde{w}_{k}^{P, \phi}\left(y_{1}-P_{1}, y_{2}+\left(y_{1}-P_{1}\right) \tan \tau-P_{2}\right) \\
& -\tilde{w}_{k}^{P, \tau}\left(y_{1}-P_{1}, y_{2}+\left(y_{1}-P_{1}\right) \tan \tau-P_{2}\right) .
\end{aligned}
$$

To estimate the first difference, we can use the Lipschitz-continuity of $\tilde{w}_{k}^{P, \phi}$. An estimate for the second difference follows by a more complicated argument: Let us introduce the functions

$$
\tilde{\theta}_{k}^{P, \phi, \tau}=\tilde{w}_{k}^{P, \phi}-\tilde{w}_{k}^{P, \tau}
$$


which are defined on our standard boundary layer-cell $\tilde{Z}=]-\frac{1}{2}, \frac{1}{2}[\times] 0, \infty[$ and can be extended periodically to $\mathbb{R} \times(0, \infty)$. The functions $\tilde{\theta}_{k}^{P, \phi, \tau}$ satisfy the following problems:

$$
\begin{aligned}
& \partial_{\tilde{y}_{i}}\left(\tilde{\mathcal{A}}_{i j}^{P, \phi}\left(\tilde{y}_{1}\right) \partial_{\tilde{y}_{j}} \tilde{\theta}_{k}^{P, \phi, \tau}(\tilde{y})\right)=\partial_{\tilde{y}_{i}}\left(\left(\tilde{\mathcal{A}}_{i j}^{P, \tau}-\tilde{\mathcal{A}}_{i j}^{P, \phi}\right) \partial_{\tilde{y}_{j}} \tilde{w}_{k}^{P, \tau}\right), \tilde{y} \in \mathbb{R} \times(0, \infty) ; \\
& \tilde{\theta}_{k}^{P, \phi, \tau}\left(\tilde{y}_{1}, 0\right)=0 ; \\
& \tilde{\theta}_{k}^{P, \phi, \tau} \text { periodic in } \tilde{y}_{1} \text { with the period } 1 ; \\
& \tilde{\theta}_{k}^{P, \phi, \tau} \text { decreases exponentially as } \tilde{y}_{n} \rightarrow \infty .
\end{aligned}
$$

By using Lemma A.1, the norm of $\tilde{\theta}_{k}^{P, \phi, \tau}$ in $W_{\gamma}(\tilde{Z})$ can be estimated by:

$$
\begin{aligned}
\left\|\tilde{\theta}_{k}^{P, \phi, \tau}\right\|_{W_{\gamma}(\tilde{Z})} \leq & \left\|\tilde{\mathcal{A}}_{i j}^{P, \tau}-\tilde{\mathcal{A}}_{i j}^{P, \phi}\right\|_{L_{\infty}(\tilde{Z})}\left\|\tilde{w}_{k}^{P, \tau}\right\|_{W_{\gamma}(\tilde{Z})} \\
= & \left\|\left(T^{t}(\tau) \mathcal{A}\left(y_{1}\left(\tilde{y}_{1}\right)\right) T(\tau)-T^{t}(\phi) \mathcal{A}\left(y_{1}\left(\tilde{y}_{1}\right)\right) T(\phi)\right)\right\|_{L_{\infty}(\tilde{Z})}\left\|\tilde{w}_{k}^{P, \tau}\right\|_{W_{\gamma}(\tilde{Z})} \\
\leq & \left\|\left(T^{t}(\tau)-T^{t}(\phi)\right) \mathcal{A}\left(y_{1}\left(\tilde{y}_{1}\right)\right) T(\tau)\right\|_{L_{\infty}(\tilde{Z})}\left\|\tilde{w}_{k}^{P, \tau}\right\|_{W_{\gamma}(\tilde{Z})} \\
& +\left\|T^{t}(\phi) \mathcal{A}\left(y_{1}\left(\tilde{y}_{1}\right)\right)(T(\tau)-T(\phi))\right\|_{L_{\infty}(\tilde{Z})}\left\|\tilde{w}_{k}^{P, \tau}\right\|_{W_{\gamma}(\tilde{Z})} \\
\leq & C|\tau-\phi|\left\|\tilde{w}_{k}^{P, \tau}\right\|_{W_{\gamma}(\tilde{Z})} .
\end{aligned}
$$

In the next step, we will derive a Hölder-estimate for the gradient of $\tilde{\theta}_{k}^{P, \phi, \tau}$. Because the right hand side of (33) is Hölder-continuous, regularity results for weak solutions imply that for $\tilde{y}^{0} \in \tilde{Z}$ with $\left|\tilde{y}_{2}^{0}\right| \geq \frac{1}{4}$ :

$$
\left\|\nabla \tilde{\theta}_{k}^{P, \phi, \tau}\right\|_{C^{0, \kappa}\left(B_{\frac{1}{16}}\left(\tilde{y}^{0}\right)\right)} \leq C\left\|\tilde{\theta}_{k}^{P, \phi, \tau}\right\|_{L_{2}\left(B_{\frac{1}{4}}\left(\tilde{y}^{0}\right)\right)}+C \sup _{i=1,2}\left[\left(\tilde{\mathcal{A}}_{i j}^{P, \tau}-\tilde{\mathcal{A}}_{i j}^{P, \phi}\right) \partial_{\tilde{y}_{j}} \tilde{w}_{k}^{P, \tau}\right]_{C^{0, \kappa}\left(B_{\frac{1}{8}}\left(\tilde{y}^{0}\right)\right)} .
$$

Since the left hand side is invariant under shifts by constants, replacing $\tilde{\theta}_{k}^{P, \phi, \tau}$ by

$$
\tilde{\theta}_{k}^{P, \phi, \tau}-\frac{1}{\left|B_{\frac{1}{4}}\left(\tilde{y}^{0}\right)\right|} \int_{B_{\frac{1}{4}}\left(\tilde{y}^{0}\right)} \tilde{\theta}_{k}^{P, \phi, \tau}
$$

and applying the Poincaré inequality yields:

$$
\begin{aligned}
\left\|\nabla \tilde{\theta}_{k}^{P, \phi, \tau}\right\|_{C^{0, \kappa}\left(B_{\frac{1}{16}}\left(\tilde{y}^{0}\right)\right)} \leq & C\left\|\nabla \tilde{\theta}_{k}^{P, \phi, \tau}\right\|_{L_{2}\left(B_{\frac{1}{4}}\left(\tilde{y}^{0}\right)\right)} \\
& +C \sup _{i=1,2}\left[\left(\tilde{\mathcal{A}}_{i j}^{P, \tau}-\tilde{\mathcal{A}}_{i j}^{P, \phi}\right) \partial_{\tilde{y}_{j}} \tilde{w}_{k}^{P, \tau}\right]_{C^{0, \kappa}\left(B_{\frac{1}{8}}\left(\tilde{y}^{0}\right)\right)}
\end{aligned}
$$

If we now use the local Hölder estimate (8) and the $W_{\gamma}$-estimate (34), we get for all $\tilde{y}^{0} \in \tilde{Z}$ with $\tilde{y}_{2}^{0} \geq \frac{1}{4}$ the following estimate of the gradient of $\tilde{\theta}_{k}^{P, \phi, \tau}$ :

$$
\begin{aligned}
\left\|\nabla \tilde{\theta}_{k}^{P, \phi, \tau}\right\|_{C^{0, \kappa}\left(B_{\frac{1}{16}}\left(\tilde{y}^{0}\right)\right)} & \leq C \mathrm{e}^{-\gamma y_{2}^{0}}\left\|\tilde{\theta}_{k}^{P, \phi, \tau}\right\|_{W_{\gamma}(\tilde{Z})}+C|\tau-\phi| \mathrm{e}^{-\gamma y_{2}^{0}}\left\|\nabla \tilde{w}_{k}^{P, \tau}\right\|_{W_{\gamma}(\tilde{Z})} \\
& \leq C|\tau-\phi| \mathrm{e}^{-\gamma \tilde{y}_{2}^{0}}
\end{aligned}
$$

For $\tilde{y}^{0}$ with $\left|\tilde{y}_{2}^{0}\right|<\frac{1}{4}$ we obtain an analogous estimate on $B_{\frac{1}{4}}\left(\tilde{y}_{1}^{0}, 0\right) \cap \tilde{Z}$. 
Integration by parts and the homogeneous boundary condition of $\tilde{\theta}_{k}^{P, \phi, \tau}$ on $\tilde{y}_{2}=0$ leads to:

$$
\left|\tilde{\theta}_{k}^{P, \phi, \tau}\left(\tilde{y}_{1}, \tilde{y}_{2}\right)\right| \leq \int_{0}^{\tilde{y}_{2}}\left|\partial_{\rho} \tilde{\theta}_{k}^{P, \phi, \tau}\left(\tilde{y}_{1}, \rho\right)\right| \mathrm{d} \rho \leq C|\tau-\phi| \int_{0}^{\tilde{y}_{2}} \mathrm{e}^{-\gamma \rho} \mathrm{d} \rho \leq C|\tau-\phi| .
$$

Now, from (32) it follows immediately that:

$$
\left|w_{k}^{P, \phi}(y)-w_{k}^{P, \tau}(y)\right| \leq C\left\{\left|y_{1}-P_{1}\right||\tan \phi-\tan \tau|+|\tau-\phi|\right\}
$$

and the the lemma is proven.

Let us now consider the domain

$$
G=\left\{(P, \phi, y) \mid P=\left(P_{1}, P_{2}\right) \in \mathbb{R}^{2}, \phi \in\right]-\phi_{g}, \phi_{g}\left[, y \in H_{P, \phi} \cap\left\{\left|y_{1}-P_{1}\right| \leq \rho\right\}\right\},
$$

and set:

$$
\Phi_{k}: G \rightarrow \mathbb{R}, \quad(P, \phi, y) \mapsto w_{k}^{P, \phi}(y) .
$$

By Theorem 4.6, Lemma 4.7, Lemma 4.8, and the triangle inequality, we have that the functions $\Phi_{k}$ are Lipschitz-continuous on $\mathrm{G}$ with a global Lipschitz constant $C_{\text {Lip }}(\rho)$. Then by Theorem A.6, we have that $\Phi_{k}$ belong to $W^{1, \infty}(G)$ and the norm of its gradient can be estimated by:

$$
\left\|\nabla \Phi_{k}\right\|_{L_{\infty}(G)} \leq C_{\operatorname{Lip}}(\rho) .
$$

Let us then return to the generalized boundary layers which are given by the composition

$$
w_{k}^{\frac{\omega(\cdot)}{\varepsilon}, \phi(\cdot)}\left(\frac{\dot{ }}{\varepsilon}\right)=\Phi_{k} \circ \tau
$$

where the function $\tau: \Omega \rightarrow G$ is defined by:

$$
\tau(x)=\left(\frac{x_{1}}{\varepsilon}, \frac{g\left(x_{1}\right)}{\varepsilon}, \phi\left(x_{1}\right), \frac{x_{1}}{\varepsilon}, \frac{x_{2}}{\varepsilon}\right) .
$$

Since $\tau$ is a $C^{2}$-diffeomorphism mapping $\Omega$ to $S \subset G$ with

$$
S=\left\{(P, \phi, y) \in G \mid P=\left(P_{1}, P_{2}\right) \in \frac{1}{\varepsilon} \partial \Omega, \phi=\phi_{P}, y_{1}=P_{1}\right\},
$$

and $\Phi_{k}$ is Lipschitz-continuous on $G$, the composition $\Phi_{k} \circ \tau$ is Lipschitz-continuous on $\Omega$. Using Theorem A.6 again, we obtain that $\Phi_{k} \circ \tau$ is differentiable almost everywhere in $\Omega$. However, for our calculations we also need an explicit representation of the partial derivatives of $\Phi_{k} \circ \tau$. We cannot get such an representation immediately because the chain rule does not apply for our situation. This is due to the fact that $\tau$ maps $\Omega$ into the submanifold $S$ of $G$, on which $\Phi_{k}$ might be not differentiable. To overcome this difficulty, we will use the following theorem:

Theorem 4.9. Let $\Omega \subset \mathbb{R}^{n}$ and $G \subset \mathbb{R}^{m}$ be two domains. We consider the functions

$$
\tau: \Omega \rightarrow \tau(\Omega) \subset G
$$

to be a $C^{1}$-diffeomorphism and

$$
\Phi: G \rightarrow \mathbb{R}
$$


to be Lipschitz-continuous. Let $\tilde{\Phi}: \Omega \rightarrow \mathbb{R}$ be the composition:

$$
\tilde{\Phi}=\Phi \circ \tau \text {. }
$$

If $\overrightarrow{\tilde{v}}$ is a vector in $\mathbb{R}^{n}$ and $x^{0} \in \Omega$ is a point where $\tilde{\Phi}$ is differentiable in the direction $\overrightarrow{\tilde{v}}$, then $\Phi$ is differentiable in $\tau\left(x^{0}\right)$ in the direction $\vec{v}=D \tau\left(x^{0}\right) \cdot \overrightarrow{\vec{v}}$ and the directional derivative is given by

$$
D_{\vec{v}} \Phi\left(\tau\left(x^{0}\right)\right)=D_{\vec{v}} \tilde{\Phi}\left(x^{0}\right) .
$$

Proof. For $h>0$ such that $x^{0}+h \overrightarrow{\tilde{v}} \in \Omega$ and $\tau\left(x^{0}\right)+h \vec{v} \in G$, let us consider the difference

$$
\Phi\left(\tau\left(x^{0}\right)+h \vec{v}\right)-\Phi(\tau(x))=\Phi\left(\tau\left(x^{0}\right)+h \vec{v}\right)-\Phi\left(\tau\left(x^{0}+h \overrightarrow{\tilde{v}}\right)\right)+\Phi\left(\tau\left(x^{0}+h \overrightarrow{\tilde{v}}\right)\right)-\Phi(\tau(x)) .
$$

For the first term, we use the Lipschitz continuity of $\Phi$ on $G$ and the fact that $\tau$ is a $C^{2}$-diffeomorphism on $\Omega$ to get:

$$
\begin{aligned}
\left|\Phi\left(\tau\left(x^{0}\right)+h \vec{v}\right)-\Phi\left(\tau\left(x^{0}+h \overrightarrow{\tilde{v}}\right)\right)\right| & \leq C_{\text {Lip }}\left|\tau\left(x^{0}\right)+h \vec{v}-\tau\left(x^{0}+h \overrightarrow{\tilde{v}}\right)\right| \\
& =C_{\text {Lip }}\left|\tau\left(x^{0}\right)+h D \tau\left(x^{0}\right) \overrightarrow{\tilde{v}}-\tau\left(x^{0}+h \overrightarrow{\tilde{v}}\right)\right| \\
& =C_{\text {Lip }} \cdot O\left(h^{2}\right)\|\tau\|_{C^{2}} \leq C h^{2} .
\end{aligned}
$$

For the second term, we use the differentiability property of $\tilde{\Phi}$ as follows:

$$
\begin{aligned}
\Phi\left(\tau\left(x^{0}+h \overrightarrow{\tilde{v}}\right)\right)-\Phi(\tau(x)) & =\tilde{\Phi}\left(x^{0}+h \overrightarrow{\tilde{v}}\right)-\tilde{\Phi}(x) \\
& =h D_{\vec{v}} \tilde{\Phi}(x)+o(h) .
\end{aligned}
$$

From the relations (40) and (41), the assertion of the theorem follows.

Theorem 4.9 now implies the following result for the weak partial derivatives of the generalized boundary layers:

Theorem 4.10. The generalized boundary layers $w_{k}^{\frac{\omega(\cdot)}{\varepsilon}, \phi(\cdot)}(\dot{\bar{\varepsilon}})$ lie in $W^{1, \infty}(\Omega)$, and the weak partial derivatives are given by:

$$
\begin{aligned}
& \partial_{x_{1}} w_{k}^{\frac{\omega\left(x_{1}\right)}{\varepsilon}, \phi\left(x_{1}\right)}\left(\frac{x}{\varepsilon}\right)=\frac{1}{\varepsilon} \partial_{y_{1}} w_{k}^{\frac{\omega\left(x_{1}\right)}{\varepsilon}, \phi\left(x_{1}\right)}\left(\frac{x}{\varepsilon}\right)+W(x) \\
& \partial_{x_{2}} w_{k}^{\frac{\omega\left(x_{1}\right)}{\varepsilon}, \phi\left(x_{1}\right)}\left(\frac{x}{\varepsilon}\right)=\frac{1}{\varepsilon} \partial_{y_{2}} w_{k}^{\frac{\omega\left(x_{1}\right)}{\varepsilon}, \phi\left(x_{1}\right)}\left(\frac{x}{\varepsilon}\right)
\end{aligned}
$$

where $W: \Omega \rightarrow \mathbb{R}$ is a measurable function with $\|W\|_{L_{\infty}(\Omega)} \leq C$.

Proof. For $x \in \Omega$, we have

$$
w_{k}^{\frac{\omega\left(x_{1}\right)}{\varepsilon}, \phi\left(x_{1}\right)}\left(\frac{x}{\varepsilon}\right)=\left(\left.\Phi_{k}\right|_{S} \circ \tau\right)(x)=: \tilde{\Phi}_{k}(x),
$$

and from our previous considerations about $\tilde{\Phi}_{k}$, it follows that the $w_{k}^{\frac{\omega(\cdot)}{\varepsilon}, \phi(\cdot)}(\dot{\bar{\varepsilon}})$ lie in $W^{1, \infty}(\Omega)$. By Theorem 4.9, the weak partial derivatives can be calculated as follows:

$$
\begin{aligned}
\partial_{x_{i}} w_{k}^{\frac{\omega\left(x_{1}\right)}{\varepsilon}, \phi\left(x_{1}\right)}\left(\frac{x}{\varepsilon}\right) & =D_{\vec{e}_{i}} \tilde{\Phi}_{k}(x)=D_{D \tau(x) \cdot \vec{e}_{i}} \Phi_{k}(\tau(x)) \\
& =\lim _{h \rightarrow 0} \frac{\Phi_{k}\left(\tau(x)+h D \tau(x) \cdot \vec{e}_{i}\right)-\Phi_{k}(\tau(x))}{h}
\end{aligned}
$$


where the vectors $D \tau(x) \cdot \vec{e}_{i}$ are given by:

$$
D \tau(x) \cdot \vec{e}_{1}=\left(\begin{array}{c}
\frac{1}{\varepsilon} \\
\frac{g^{\prime}\left(x_{1}\right)}{\varepsilon} \\
\phi^{\prime}\left(x_{1}\right) \\
\frac{1}{\varepsilon} \\
0
\end{array}\right) \text { and } D \tau(x) \cdot \vec{e}_{2}=\left(\begin{array}{l}
0 \\
0 \\
0 \\
0 \\
1
\end{array}\right)
$$

To get the explicit formulas (42) and (43), we use the definition of the functions $\Phi_{k}$. Thus, let us first consider the difference-quotient corresponding to the partial derivative with respect to $x_{1}$ :

$$
\begin{aligned}
\frac{1}{h}\left(\Phi_{k}(\tau(x)+\right. & \left.\left.h D \tau(x) \cdot \vec{e}_{1}\right)-\Phi_{k}(\tau(x))\right) \\
= & \frac{1}{h}\left(w_{k}^{\frac{\omega\left(x_{1}\right)}{\varepsilon}+h\left(\frac{1}{\varepsilon}, \frac{g^{\prime}\left(x_{1}\right)}{\varepsilon}\right), \phi\left(x_{1}\right)+h \phi^{\prime}\left(x_{1}\right)}\left(\frac{x_{1}}{\varepsilon}+\frac{h}{\varepsilon}, \frac{x_{2}}{\varepsilon}\right)\right. \\
& \left.-w_{k}^{\frac{\omega\left(x_{1}\right)}{\varepsilon}, \phi\left(x_{1}\right)}\left(\frac{x_{1}}{\varepsilon}, \frac{x_{2}}{\varepsilon}\right)\right) \\
= & \frac{1}{h}\left(w_{k}^{\frac{\omega\left(x_{1}\right)}{\varepsilon}, \phi\left(x_{1}\right)}\left(\frac{x_{1}}{\varepsilon}+\frac{h}{\varepsilon}, \frac{x_{2}}{\varepsilon}\right)-w_{k}^{\frac{\omega\left(x_{1}\right)}{\varepsilon}, \phi\left(x_{1}\right)}\left(\frac{x_{1}}{\varepsilon}, \frac{x_{2}}{\varepsilon}\right)\right) \\
& +\frac{1}{h}\left(w_{k}^{\frac{\omega\left(x_{1}\right)}{\varepsilon}+h\left(\frac{1}{\varepsilon}, \frac{g^{\prime}\left(x_{1}\right)}{\varepsilon}\right), \phi\left(x_{1}\right)}\left(\frac{x_{1}}{\varepsilon}+\frac{h}{\varepsilon}, \frac{x_{2}}{\varepsilon}\right)\right. \\
& \left.-w_{k}^{\frac{\omega\left(x_{1}\right)}{\varepsilon}, \phi\left(x_{1}\right)}\left(\frac{x_{1}}{\varepsilon}+\frac{h}{\varepsilon}, \frac{x_{2}}{\varepsilon}\right)\right) \\
& +\frac{1}{h}\left(w_{k}^{\frac{\omega\left(x_{1}\right)}{\varepsilon}+h\left(\frac{1}{\varepsilon}, \frac{g^{\prime}\left(x_{1}\right)}{\varepsilon}\right), \phi\left(x_{1}\right)+h \phi^{\prime}\left(x_{1}\right)}\left(\frac{x_{1}}{\varepsilon}+\frac{h}{\varepsilon}, \frac{x_{2}}{\varepsilon}\right)\right. \\
& \left.-w_{k}^{\frac{\omega\left(x_{1}\right)}{\varepsilon}+h\left(\frac{1}{\varepsilon}, \frac{g^{\prime}\left(x_{1}\right)}{\varepsilon}\right), \phi\left(x_{1}\right)}\left(\frac{x_{1}}{\varepsilon}+\frac{h}{\varepsilon}, \frac{x_{2}}{\varepsilon}\right)\right) .
\end{aligned}
$$

For $h \rightarrow 0$, the first difference (45) tends to

$$
\frac{1}{\varepsilon} \partial_{y_{1}} w_{k}^{\frac{\omega\left(x_{1}\right)}{\varepsilon}, \phi\left(x_{1}\right)}\left(\frac{x_{1}}{\varepsilon}, \frac{x_{2}}{\varepsilon}\right),
$$

due to the $C^{1, \kappa}$-regularity of the generalized boundary layers. The second difference is zero because the point $\frac{\omega\left(x_{1}\right)}{\varepsilon}+h\left(\frac{1}{\varepsilon}, \frac{g^{\prime}\left(x_{1}\right)}{\varepsilon}\right)$ lies on the tangent $T_{\frac{\omega\left(x_{1}\right)}{\varepsilon}, \phi\left(x_{1}\right)}$ and the parameters $\frac{\omega\left(x_{1}\right)}{\varepsilon}+h\left(\frac{1}{\varepsilon}, \frac{g^{\prime}\left(x_{1}\right)}{\varepsilon}\right), \phi\left(x_{1}\right)$ determine the same generalized boundary layers as $\frac{\omega\left(x_{1}\right)}{\varepsilon}, \phi\left(x_{1}\right)$. For the third difference (47), we use the Lipschitz-continuity of the functions $\Phi_{k}$ to estimate the absolute value of this difference by

$$
C_{\text {Lip }}\left|\phi^{\prime}\left(x_{1}\right)\right|
$$

These arguments imply the formula (42). The formula (43) for the partial derivatives of the generalized boundary layers with respect to $x_{2}$ follows immediately from (44) and the $C^{1, \kappa}$-regularity of the generalized boundary layers. 


\subsection{Error estimates}

In this section, we will show that for domains $\Omega$ given by (22) in the case of a layered medium the approximation

$$
u_{\varepsilon}^{a p p}=u^{0}+\varepsilon \partial_{k} u^{0} w^{k}\left(\frac{\dot{\varepsilon}}{\varepsilon}\right)+w^{\varepsilon}
$$

with $w^{\varepsilon}$ defined in (30) leads to an error estimate of order $\varepsilon$ in the energy norm. This is an extension to domains with curved boundaries of the results given in $[6,7,9,14,19,20]$ for the half space.

Theorem 4.11. Let the domain $\Omega$ be given by (22), and let $u^{\varepsilon}$ be the solution of problem (24) where the coefficients $\mathcal{A}_{i j}^{\varepsilon}$ are given by $\mathcal{A}: \mathbb{R} \rightarrow \mathbb{R}^{2 \times 2}$, with $\mathcal{A}_{i j}\left(y_{1}\right)$ periodic in $y_{1}$ with period 1 , bounded, and strongly elliptic, satisfying the additional regularity assumption $\mathcal{A} \in C^{0, \kappa}\left(\mathbb{R}, \mathbb{R}^{2 \times 2}\right)$ with $0<\kappa<1$. If the solution $u^{0}$ of the homogenized problem satisfies $\left\|\nabla^{2} u^{0}\right\|_{L_{2}(\Omega)} \leq C$, then the following estimate holds:

$$
\left\|u^{\varepsilon}-u_{\varepsilon}^{\mathrm{app}}\right\|_{\dot{H}^{1}(\Omega)} \leq C \varepsilon\left(\left\|\nabla u^{0}\right\|_{L_{2}(\Omega)}+\left\|\nabla^{2} u^{0}\right\|_{L_{2}(\Omega)}\right)
$$

To prove this theorem, we need additional results which we formulate in the next lemmata.

Lemma 4.12. Let the coefficients matrix $\mathcal{A}_{i j}$ be given as in Theorem 4.11. For $k=1,2$ we consider the vector $\vec{h}_{k}^{P, \phi}: H_{P, \phi} \rightarrow \mathbb{R}^{2}$ given by:

$$
h_{k, i}^{P, \phi}=\mathcal{A}_{i j} \partial_{y_{j}} w_{k}^{P, \phi}, i=1,2
$$

Then there exist skew-symmetric tensors $\left(\beta_{k}^{P, \phi}\right): H_{P, \phi} \rightarrow \mathbb{R}^{2 \times 2}$ with the following properties:

(a) $\beta_{k, l i}^{P, \phi} \in C^{1, \kappa}\left(H_{P, \phi}\right) \cap L_{\infty}\left(H_{P, \phi}\right), l, i=1,2$;

(b) $\partial_{l} \beta_{k, l i}^{P, \phi}=h_{k, i}^{P, \phi}, i=1,2$;

(c) $\beta_{k}^{P, \phi}$ is periodic in the direction parallel to $T_{P, \phi}$;

(d) $\nabla \beta_{k}^{P, \phi}$ decreases exponentially normal to $T_{P, \phi}$.

Proof. For each $k=1,2$, we construct the matrix $\left(\beta_{k}^{P, \phi}\right)$ by help of the vector $\vec{\zeta}_{k}^{P, \phi}$ defined as the solution of the following system:

$$
\begin{array}{ll}
\Delta \vec{\zeta}_{k}^{P, \phi}=\vec{h}_{k}^{P, \phi}, & y \in H_{P, \phi} \\
\vec{\zeta}_{k}^{P, \phi} \cdot \vec{t}=0, & y \in T_{P, \phi} \\
\frac{\partial}{\partial \vec{n}}\left(\vec{\zeta}_{k}^{P, \phi} \cdot \vec{n}\right)=0, \quad y \in T_{P, \phi} \\
\vec{\zeta}_{k}^{P, \phi} \text { periodic parallel to the boundary } \\
\nabla \vec{\zeta}_{k}^{P, \phi} \text { decreases exponentially normal to the boundary }
\end{array}
$$

where the vectors $\vec{t}, \vec{n}$ are given by: $\vec{t}=\vec{t}(\phi)=(\cos \phi, \sin \phi)$ and $\vec{n}=\vec{n}(\phi)=(-\sin \phi, \cos \phi)$. The system above can be decoupled if we write

$$
\vec{\zeta}_{k}^{P, \phi}=\left(\vec{\zeta}_{k}^{P, \phi} \cdot \vec{t}\right) \vec{t}+\left(\vec{\zeta}_{k}^{P, \phi} \cdot \vec{n}\right) \vec{n}=\zeta_{k, t}^{P, \phi} \vec{t}+\zeta_{k, n}^{P, \phi} \vec{n}
$$


and consider the problems for $\zeta_{k, t}^{P, \phi}$ and $\zeta_{k, n}^{P, \phi}$ :

$$
\begin{aligned}
& \Delta \zeta_{k, t}^{P, \phi}=\vec{h}_{k}^{P, \phi} \cdot \vec{t}, \quad y \in H_{P, \phi} \\
& \zeta_{k, t}^{P, \phi}=0, \quad y \in T_{P, \phi} \\
& \zeta_{k, t}^{P, \phi} \text { periodic parallel to the boundary } \\
& \nabla \zeta_{k, t}^{P, \phi} \text { decreases exponentially normal to the boundary }
\end{aligned}
$$

and

$$
\begin{aligned}
& \Delta \zeta_{k, n}^{P, \phi}=\vec{h}_{k}^{P, \phi} \cdot \vec{n}, \quad y \in H_{P, \phi} \\
& \frac{\partial}{\partial \vec{n}}\left(\zeta_{k, n}^{P, \phi}\right)=0, \quad y \in T_{P, \phi} \\
& \zeta_{k, n}^{P, \phi} \text { periodic parallel to the boundary } \\
& \nabla \zeta_{k, n}^{P, \phi} \text { decreases exponentially normal to the boundary. }
\end{aligned}
$$

Now by a simple rotation we get the situation of Lemma 2.6. By also using the $C^{0, \kappa}$ regularity of the vector $\vec{h}_{k}^{P, \phi}$, we have that the solutions $\zeta_{k, t}^{P, \phi}$ and $\zeta_{k, n}^{P, \phi}$ exist and are uniquely determined in $C^{2, \kappa}\left(H_{P, \phi}\right) \cap W^{1, \infty}\left(H_{P, \phi}\right)$.

Using the vectors $\vec{\zeta}_{k}^{P, \phi}$, we define the tensor $\left(\beta_{k}^{P, \phi}\right)$ by the following formulas:

$$
\beta_{k, l i}^{P, \phi}(y):=\partial_{y_{l}} \zeta_{k, i}^{P, \phi}(y)-\partial_{y_{i}} \zeta_{k, l}^{P, \phi}(y), l, i=1,2 .
$$

Then $\left(\beta_{k}^{P, \phi}\right)$ is skew-symmetric, and due to the regularity of $\zeta_{k, i}^{P, \phi}$ it belongs to $\left(C^{1, \kappa}\left(H_{P, \phi}\right)\right)^{2 \times 2} \cap\left(L_{\infty}\left(H_{P, \phi}\right)\right)^{2 \times 2}$. The relation (b) can be proven analogously to relation (16) form Lemma 2.6 .

In the proof of Theorem 4.11, we will deal with matrices $\left(\beta_{k}^{P, \phi}(y)\right)$ of the form $\left(\beta_{k}^{P(x), \phi(x)}(y(x))\right)$, for $x \in \Omega$; and we have to consider derivatives of the matrix elements with respect to $x$. Therefore, we have to study the regularity of $\left(\beta_{k}^{P, \phi}\right)$ with respect to the parameters $(P, \phi)$. The regularity relative to $y$ is given by Lemma 4.12. We had to deal with a similar problem when we studied the regularity of the boundary corrector $w^{\varepsilon}$. Using the same techniques, we obtain the following analogues to Lemma 4.7 and Lemma 4.8.

Lemma 4.13. Let $\mathcal{A}_{i j} \in C^{0, \kappa}(\mathbb{R})$ with $\kappa \in(0,1), P, Q$ be two neighboring points in $\mathbb{R}^{2}$ and $\left.\phi \in\right]-\phi_{g}$, $\phi_{g}[$. Then for each $y \in H_{P, \phi} \cap H_{Q, \phi}$ the following estimate holds:

$$
\left|\partial_{y_{l}} \zeta_{k, i}^{P, \phi}(y)-\partial_{y_{l}} \zeta_{k, i}^{Q, \phi}(y)\right| \leq C\|P-Q\|
$$

Lemma 4.14. Let $\mathcal{A}_{i j} \in C^{0, \kappa}(\mathbb{R})$ with $\kappa \in(0,1), P \in \mathbb{R}^{2}$ and $\phi, \tau$ be two neighboring angles in $]-\phi_{g}$, $\phi_{g}[$. Then for each $y \in H_{P, \phi} \cap H_{P, \tau}$ the following estimate holds:

$$
\left|\partial_{y_{l}} \zeta_{k, i}^{P, \phi}(y)-\partial_{y_{l}} \zeta_{k, i}^{P, \tau}(y)\right| \leq C\left|y_{1}-P_{1}\right||\phi-\tau|+C|\phi-\tau| .
$$

If we now regard $\left(\beta_{k}^{P, \phi}\right)$ defined by (53) as matrix-valued functions depending on the variables $(P, \phi, y)$, then, by the lemmas above, it follows that they are uniformly Lipschitz-continuous for $(P, \phi, y)$ belonging to the domain

$$
G=\left\{(P, \phi, y) \mid P=\left(P_{1}, P_{2}\right) \in \mathbb{R}^{2}, \phi \in\right]-\phi_{g}, \phi_{g}\left[, y \in H_{P, \phi} \cap\left\{\left|y_{1}-P_{1}\right| \leq \rho\right\}\right\},
$$

where $\rho$ is an arbitrary positive constant. By Theorem A.6, we have that $\left(\beta_{k}^{P, \phi}\right)$ belongs to $\left(W^{1, \infty}(G)\right)^{2 \times 2}$ and

$$
\left\|\nabla \beta_{k, l i}^{P, \phi}\right\|_{L_{\infty}(G)} \leq C_{\text {Lip }}(\rho) .
$$


Now the same arguments as in the study of the boundary corrector $w^{\varepsilon}$ lead to the following theorem concerning the differentiability of the matrices $\left(\beta_{k}^{\frac{\omega\left(x_{1}\right)}{\varepsilon}, \phi\left(x_{1}\right)}\left(\frac{x}{\varepsilon}\right)\right)$ with respect to $x$ :

Theorem 4.15. The functions $\beta_{k, l i}^{\frac{\omega(\cdot)}{\varepsilon}, \phi(\cdot)}(\dot{\bar{\varepsilon}})$ lie in $W^{1, \infty}(\Omega)$, and the weak partial derivatives are given by:

$$
\begin{aligned}
& \partial_{x_{1}} \beta_{k, l i}^{\frac{\omega\left(x_{1}\right)}{\varepsilon}, \phi\left(x_{1}\right)}\left(\frac{x}{\varepsilon}\right)=\frac{1}{\varepsilon} \partial_{y_{1}} \beta_{k, l i}^{\frac{\omega\left(x_{1}\right)}{\varepsilon}, \phi\left(x_{1}\right)}\left(\frac{x}{\varepsilon}\right)+B(x) \\
& \partial_{x_{2}} \beta_{k, l i}^{\frac{\omega\left(x_{1}\right)}{\varepsilon}, \phi\left(x_{1}\right)}\left(\frac{x}{\varepsilon}\right)=\frac{1}{\varepsilon} \partial_{y_{2}} \beta_{k, l i}^{\frac{\omega\left(x_{1}\right)}{\varepsilon}, \phi\left(x_{1}\right)}\left(\frac{x}{\varepsilon}\right)
\end{aligned}
$$

where $B: \Omega \rightarrow \mathbb{R}$ is a measurable function with $\|B\|_{L_{\infty}(\Omega)} \leq C$.

Now we can start with:

Proof of Theorem 4.11. We will proceed as in the proof of Theorem 2.5. This time, however, we have to deal with additional difficulties since the generalized boundary layers depend on the boundary parameters. Thus, for all testfunctions $\varphi \in \dot{H}_{0}^{1}(\Omega)$, we have:

$$
\begin{aligned}
& \int_{\Omega} \mathcal{A}_{i j}^{\varepsilon}\left(x_{1}\right) \partial_{j}\left(u^{\varepsilon}(x)-u^{0}(x)-\varepsilon \partial_{k} u^{0}(x)\left(w^{k}\left(\frac{x}{\varepsilon}\right)+w_{k}^{\frac{\omega\left(x_{1}\right)}{\varepsilon}, \phi\left(x_{1}\right)}\left(\frac{x}{\varepsilon}\right)\right)\right) \partial_{i} \varphi \\
= & \int_{\Omega}\left(\mathcal{A}_{i j}^{0} \partial_{j} u^{0}(x)-\mathcal{A}_{i j}^{\varepsilon}\left(x_{1}\right) \partial_{j} u^{0}(x)-\mathcal{A}_{i j}^{\varepsilon}\left(x_{1}\right) \varepsilon \partial_{j}\left(\partial_{k} u^{0}(x) w^{k}\left(\frac{x}{\varepsilon}\right)\right)\right) \partial_{i} \varphi(x) \mathrm{d} x \\
& -\int_{\Omega} \mathcal{A}_{i j}^{\varepsilon}\left(x_{1}\right) \varepsilon \partial_{j}\left(\partial_{k} u^{0}(x) w_{k}^{\frac{\omega\left(x_{1}\right)}{\varepsilon}, \phi\left(x_{1}\right)}\left(\frac{x}{\varepsilon}\right)\right) \partial_{i} \varphi(x) \mathrm{d} x \\
= & \int_{\Omega}\left(\mathcal{A}_{i j}^{0}-\mathcal{A}_{i j}^{\varepsilon}\left(x_{1}\right)-\mathcal{A}_{i k}^{\varepsilon}\left(x_{1}\right) \partial_{y_{k}} w^{j}\left(\frac{x}{\varepsilon}\right)\right) \partial_{j} u^{0}(x) \partial_{i} \varphi(x) \mathrm{d} x \\
& -\varepsilon \int_{\Omega} \mathcal{A}_{i j}^{\varepsilon}\left(x_{1}\right) \partial_{j} w_{k}^{\frac{\omega\left(x_{1}\right)}{\varepsilon}, \phi\left(x_{1}\right)}\left(\frac{x}{\varepsilon}\right) \partial_{k} u^{0}(x) \partial_{i} \varphi(x) \mathrm{d} x \\
& -\varepsilon \int_{\Omega} \mathcal{A}_{i j}^{\varepsilon}\left(x_{1}\right) \partial_{j k} u^{0}(x)\left(w^{k}\left(\frac{x}{\varepsilon}\right)+w_{k}^{\frac{\omega\left(x_{1}\right)}{\varepsilon}, \phi\left(x_{1}\right)}\left(\frac{x}{\varepsilon}\right)\right) \partial_{i} \varphi(x) \mathrm{d} x .
\end{aligned}
$$

The first term on the right hand side of (55) can be treated like in Theorem 1.3 to obtain:

$$
\int_{\Omega}\left(\mathcal{A}_{i j}^{0}-\mathcal{A}_{i j}^{\varepsilon}\left(x_{1}\right)-\mathcal{A}_{i k}^{\varepsilon}\left(x_{1}\right) \partial_{y_{k}} w^{j}\left(\frac{x}{\varepsilon}\right)\right) \partial_{j} u^{0}(x) \partial_{i} \varphi(x) \mathrm{d} x \leq C \varepsilon\left\|\nabla^{2} u^{0}\right\|_{L_{2}(\Omega)}\|\nabla \varphi\|_{L_{2}(\Omega)}+o(R) .
$$

For the second term, we first use the results of Theorem 4.10 to write it in the following way:

$$
\begin{aligned}
\varepsilon \int_{\Omega} \mathcal{A}_{i j}\left(\frac{x_{1}}{\varepsilon}\right) \partial_{j} w_{k}^{\frac{\omega\left(x_{1}\right)}{\varepsilon}, \phi\left(x_{1}\right)}\left(\frac{x}{\varepsilon}\right) \partial_{k} u^{0}(x) \partial_{i} \varphi(x) \mathrm{d} x= & \int_{\Omega} \mathcal{A}_{i j}\left(\frac{x_{1}}{\varepsilon}\right) \partial_{y_{j}} w_{k}^{\frac{\omega\left(x_{1}\right)}{\varepsilon}, \phi\left(x_{1}\right)}\left(\frac{x}{\varepsilon}\right) \partial_{k} u^{0}(x) \partial_{i} \varphi(x) \mathrm{d} x \\
& +O\left(\varepsilon\left\|\nabla u^{0}\right\|_{L_{2}(\Omega)}\|\nabla \varphi\|_{L_{2}(\Omega)}\right) .
\end{aligned}
$$

Lemma 4.12 then yields the existence of a skew-symmetric tensor $\left(\beta_{k}^{\frac{\omega\left(x_{1}\right)}{\varepsilon}, \phi\left(x_{1}\right)}\right) \in\left(C^{1, \kappa}\left(H_{\frac{\omega\left(x_{1}\right)}{\varepsilon}, \phi\left(x_{1}\right)}\right)\right)^{2 \times 2} \cap$ $\left(L_{\infty}\left(H_{\frac{\omega\left(x_{1}\right)}{\varepsilon}, \phi\left(x_{1}\right)}\right)\right)^{2 \times 2}$ such that

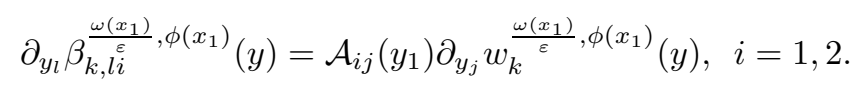


Therefore, the second term in (55) becomes:

$$
\begin{aligned}
& \varepsilon \int_{\Omega} \mathcal{A}_{i j}\left(\frac{x_{1}}{\varepsilon}\right) \partial_{j} w_{k}^{\frac{\omega\left(x_{1}\right)}{\varepsilon}, \phi\left(x_{1}\right)}\left(\frac{x}{\varepsilon}\right) \partial_{k} u^{0}(x) \partial_{i} \varphi(x) \mathrm{d} x \\
& =\int_{\Omega \cap B_{R}} \partial_{y_{l}} \beta_{k, l i}^{\frac{\omega\left(x_{1}\right)}{\varepsilon}, \phi\left(x_{1}\right)}\left(\frac{x}{\varepsilon}\right) \partial_{k} u^{0}(x) \partial_{i} \varphi(x) \mathrm{d} x \\
& +\int_{\Omega \backslash B_{R}} \mathcal{A}_{i j}\left(\frac{x_{1}}{\varepsilon}\right) \partial_{y_{j}} w_{k}^{\frac{\omega\left(x_{1}\right)}{\varepsilon}, \phi\left(x_{1}\right)}\left(\frac{x}{\varepsilon}\right) \partial_{k} u^{0}(x) \partial_{i} \varphi(x) \mathrm{d} x \\
& +O\left(\varepsilon\left\|\nabla u^{0}\right\|_{L_{2}(\Omega)}\|\nabla \varphi\|_{L_{2}(\Omega)}\right) .
\end{aligned}
$$

For the first term on the right hand side, we now use the result of Theorem 4.15 to write $\partial_{y_{l}} \beta_{k, l i}^{\frac{\omega\left(x_{1}\right)}{\varepsilon}, \phi\left(x_{1}\right)}$ in terms of derivatives with respect to $x$. The second term tends to zero for $R \rightarrow \infty$ due to the regularity of the homogenized solution $u^{0}$. Thus we have:

$$
\begin{aligned}
\varepsilon \int_{\Omega} \mathcal{A}_{i j}\left(\frac{x_{1}}{\varepsilon}\right) \partial_{j} w_{k}^{\frac{\omega\left(x_{1}\right)}{\varepsilon}, \phi\left(x_{1}\right)}\left(\frac{x}{\varepsilon}\right) \partial_{k} u^{0}(x) \partial_{i} \varphi(x) \mathrm{d} x & \\
= & \varepsilon \int_{\Omega \cap B_{R}}\left(\partial_{x_{l}} \beta_{k, l i}^{\frac{\omega\left(x_{1}\right)}{\varepsilon}, \phi\left(x_{1}\right)}\left(\frac{x}{\varepsilon}\right)+B(x)\right) \partial_{k} u^{0}(x) \partial_{i} \varphi(x) \mathrm{d} x \\
& +O\left(\varepsilon\left\|\nabla u^{0}\right\|_{L_{2}(\Omega)}\|\nabla \varphi\|_{L_{2}(\Omega)}\right)+o(R) .
\end{aligned}
$$

What remains is the analysis of the term:

$$
\begin{aligned}
\varepsilon \int_{\Omega \cap B_{R}} \partial_{x_{l}} \beta_{k, l i}^{\frac{\omega\left(x_{1}\right)}{\varepsilon}, \phi\left(x_{1}\right)}\left(\frac{x}{\varepsilon}\right) \partial_{k} u^{0}(x) \partial_{i} \varphi(x) \mathrm{d} x \\
=\quad \varepsilon \int_{\Omega \cap B_{R}} \partial_{x_{l}}\left(\beta_{k, l i}^{\frac{\omega\left(x_{1}\right)}{\varepsilon}, \phi\left(x_{1}\right)}\left(\frac{x}{\varepsilon}\right) \partial_{k} u^{0}(x)\right) \partial_{i} \varphi(x) \mathrm{d} x \\
\quad-\varepsilon \int_{\Omega \cap B_{R}} \beta_{k, l i}^{\frac{\omega\left(x_{1}\right)}{\varepsilon}, \phi\left(x_{1}\right)}\left(\frac{x}{\varepsilon}\right) \partial_{l} \partial_{k} u^{0}(x) \partial_{i} \varphi(x) \mathrm{d} x
\end{aligned}
$$

Because of the regularity of $u^{0}$ and the $L_{\infty}$-boundedness of $\beta_{k, l}^{\frac{\omega\left(x_{1}\right)}{\ell}, \phi\left(x_{1}\right)}$, the second term has the form $O\left(\varepsilon\left\|\nabla^{2} u^{0}\right\|_{L_{2}(\Omega)}\|\nabla \varphi\|_{L_{2}(\Omega)}\right)$. Integrating by parts in the first integral for $\varphi \in C^{\infty}(\Omega) \cap \dot{H}_{0}^{1}(\Omega)$ with $\operatorname{supp}(\varphi) \cap$ $\partial \Omega=\emptyset$, we get:

$$
\begin{aligned}
& \varepsilon \int_{\Omega \cap B_{R}} \partial_{x_{l}}\left(\beta_{k, l i}^{\frac{\omega\left(x_{1}\right)}{\varepsilon}, \phi\left(x_{1}\right)}\left(\frac{x}{\varepsilon}\right) \partial_{k} u^{0}(x)\right) \partial_{i} \varphi(x) \mathrm{d} x \\
&= \varepsilon \int_{\Omega \cap B_{R}} \beta_{k, l i}^{\frac{\omega\left(x_{1}\right)}{\varepsilon}, \phi\left(x_{1}\right)}\left(\frac{x}{\varepsilon}\right) \partial_{k} u^{0}(x) \partial_{l i} \varphi(x) \mathrm{d} x \\
& \quad-\varepsilon \int_{\Omega \cap \partial B_{R}} \beta_{k, l i}^{\frac{\omega\left(x_{1}\right)}{\varepsilon}, \phi\left(x_{1}\right)}\left(\frac{x}{\varepsilon}\right) \partial_{k} u^{0}(x) \partial_{i} \varphi(x) \nu_{l}(x) \mathrm{d} \sigma_{x} .
\end{aligned}
$$

The first term is zero because of the skew-symmetry of $\beta_{k, l i}^{\frac{\omega\left(x_{1}\right)}{\varepsilon}, \phi\left(x_{1}\right)}$, and the second term tends to zero for $R \rightarrow \infty$.

The third term on the right-hand side of (55) has the form $O\left(\varepsilon\left\|\nabla^{2} u^{0}\right\|_{L_{2}(\Omega)}\|\nabla \varphi\|_{L_{2}(\Omega)}\right)$, due to the $L_{\infty^{-}}$ boundedness of the cell-solutions $w^{k}$ and of the boundary correctors $w_{k}^{\varepsilon, b l}$. 
By an argument analogous to the one in the proof of Theorem 2.5, we have that the subspace $\left\{\varphi \in C^{\infty}(\bar{\Omega}) \mid\right.$ $\left.\|\nabla \varphi\|_{L_{2}(\Omega)} \leq C, \operatorname{supp}(\varphi) \cap \partial \Omega=\emptyset\right\}$ is dense in $\dot{H}_{0}^{1}(\Omega)$ with respect to the norm $\|\cdot\|_{\dot{H}^{1}(\Omega)}$. Thus, taking

$$
\varphi=u^{\varepsilon}-\left(u^{0}+\varepsilon \partial_{k} u^{0} w^{k}\left(\frac{\dot{\varepsilon}}{\varepsilon}\right)+w^{\varepsilon}\right)
$$

in (55), for $R \rightarrow \infty$, and using the ellipticity of the coefficients $\mathcal{A}_{i j}^{\varepsilon}$, we get the energy-estimate (49) for the difference $u^{\varepsilon}-u_{\varepsilon}^{\text {app }}$.

\section{Appendix A}

\section{A.1. Tartar's Lemma}

Lemma A.1. Let $(V,|\cdot|)$ and $\left(V_{0},|\cdot|_{0}\right)$ be two Hilbert spaces on $\mathbb{R}$, such that $V_{0} \subset V$ with continuous injection. We are given a continuous bilinear form $a(\cdot, \cdot)$ on $V \times V_{0}$, and a linear, continuous operator $M \in \mathcal{L}\left(V, V_{0}\right)$ such that $M$ maps $V$ onto $V_{0}$.

If there exists a $\mu>0$ such that $a(u, M u) \geq \mu|u|^{2}, \forall u \in V$ then $\forall f \in V_{0}^{\prime}$, there exists a unique $u_{0} \in V$ such that

$$
a\left(u_{0}, v\right)=\langle f, v\rangle, \quad \forall v \in V_{0}
$$

and

$$
\left|u_{0}\right| \leq C\|f\|_{V_{0}^{\prime}}
$$

Proof. See [14].

\section{A.2. Local bounds for weak solutions}

Let us consider the equation:

$$
L u:=-\partial_{i}\left(\mathcal{A}_{i j}(y) \partial_{j} u(y)\right)=g+\partial_{i} f_{i}
$$

on the domain $\Omega \subset \mathbb{R}^{n}$, with the operator L having strictly elliptic and bounded coefficients, and suppose that $f_{i} \in L_{q}(\Omega)$ and $g \in L_{\frac{q}{2}}(\Omega)$, for some $q>n$. Then we can formulate the following results:

Theorem A.2 (Inner estimates). If $u \in W^{1,2}(\Omega)$ is a weak solution of (56), then for any ball $B_{2 R}(y) \subset \Omega$ and $p>1$ we have:

$$
\sup _{B_{R}(y)}|u| \leq C\left(R^{-\frac{n}{p}}\|u\|_{L_{p}\left(B_{2 R}\right)}+R^{1-\frac{n}{q}}\|f\|_{L_{q}\left(B_{2 R}\right)}+R^{2\left(1-\frac{n}{q}\right)}\|g\|_{L_{\frac{q}{2}\left(B_{2 R}\right)}}\right) .
$$

Proof. See [11], Chapter 8, Theorem 8.17.

Theorem A.3 (Estimates at the boundary). If $u \in W^{1,2}(\Omega)$ is a subsolution of equation(56) in $\Omega$, then we have for any $y \in \mathbb{R}^{n}, R>0$ and $p>1$,

$$
\sup _{B_{R}(y)} u_{M}^{+} \leq C\left(R^{-\frac{n}{p}}\left\|u_{M}^{+}\right\|_{L_{p}\left(B_{2 R}\right)}+R^{1-\frac{n}{q}}\|f\|_{L_{q}\left(B_{2 R}\right)}+R^{2\left(1-\frac{n}{q}\right)}\|g\|_{L_{\frac{q}{2}\left(B_{2 R}\right)}}\right)
$$


where

$$
\begin{aligned}
& M=\sup _{\partial \Omega \cap B_{2 R}} u^{+} \\
& u_{M}^{+}(y)= \begin{cases}\sup \{u(y), M\}, & y \in \Omega, \\
M, & y \notin \Omega .\end{cases}
\end{aligned}
$$

Proof. See [11], Chapter 8, Theorem 8.25.

Theorem A.4 (Hölder estimates at the boundary). Let the domain $\Omega$ satisfy an exterior cone condition at a point $y_{0} \in \partial \Omega$ and $0<R<R_{0}$. If $u \in W^{1,2}(\Omega)$ is a solution of equation (56) in $\Omega$ with homogeneous right hand side, such that $u$ vanishes on $\partial \Omega \cap B_{\sqrt{R R_{0}}}\left(y_{0}\right)$, then there exists $0<\kappa<1$ with:

$$
\sup _{\Omega \cap B_{R}\left(y_{0}\right)} u-\inf _{\Omega \cap B_{R}\left(y_{0}\right)} u \leq C\left[\frac{R}{R_{0}}\right]^{\kappa} \sup _{\Omega \cap B_{R_{0}}\left(y_{0}\right)}|u|,
$$

and $C=C\left(n, \frac{\Lambda}{\lambda}\right)$.

Proof. See [11], Chapter 8, Theorem 8.27.

\section{A.3. The size of the neighborhoods in the Inverse Mapping Theorem}

Theorem A.5. Let $U$ be an open subset of $\mathbb{R}^{n}, f: U \rightarrow \mathbb{R}^{n}$ be a map of class $C^{k}, k \geq 2$ and $x_{0} \in U$ with $D f\left(x^{0}\right)$ a linear isomorphism. We denote:

$$
L=\left\|D f\left(x^{0}\right)\right\| \text { and } M=\left\|D f\left(x^{0}\right)^{-1}\right\| .
$$

Assume

$$
\left\|D^{2} f(x)\right\| \leq K, \text { for }\left\|x-x_{0}\right\| \leq R \text { and } B_{R}\left(x_{0}\right) \subset U \text {. }
$$

Let

$$
P=\min \left\{\frac{1}{2 K M}, R\right\}, Q=\min \left\{\frac{1}{2 N L}, \frac{P}{M}, P\right\}, S=\min \left\{\frac{1}{2 K M}, \frac{Q}{2 L}, Q\right\} .
$$

Here $N=8 M^{3} K$. Then $\left.f\right|_{B_{\frac{Q}{2 L}}\left(x_{0}\right)}: B_{\frac{Q}{2 L}}\left(x_{0}\right) \rightarrow B_{\frac{S}{2 M}}\left(f\left(x_{0}\right)\right)$ is a $C^{k}$-diffeomorphism.

Proof. See [2].

\section{A.4. A Rademacher-type theorem}

Theorem A.6. Let $\Omega$ be a domain in $\mathbb{R}^{n}$ and $f \in C^{0,1}(\Omega)$. Then the classical partial derivatives $\partial f / \partial x_{i}, i=1, \ldots, n$, exist almost everywhere in $\Omega$, they are measurable and bounded,

$$
\left\|\frac{\partial f}{\partial x_{i}}\right\|_{L_{\infty}(\Omega)} \leq\|f\|_{C^{0,1}(\Omega)}
$$

and they coincide with the weak derivatives.

Proof. See [22]. 


\section{REFERENCES}

[1] R.A. Adams, Sobolev Spaces. Academic Press, New York, San Francisco, London (1975).

[2] R. Abraham, J.E. Marsden and T. Ratiu, Manifolds, tensor analysis, and applications. 2nd edn. Appl. Math. Sci. 75 SpringerVerlag, New York (1988).

[3] G. Allaire, Homogenization and two-scale convergence. SIAM J. Math. Anal. 23 (1992) 1482-1518.

[4] G. Allaire and M. Amar, Boundary layer tails in periodic homogenization. ESAIM: COCV 4 (1999) 209-243.

[5] I. Babuska, Solution of interface problems by homogenization I. SIAM J. Math. Anal. 7 (1976) 603-634.

[6] I. Babuska, Solution of interface problems by homogenization II. SIAM J. Math. Anal. 7 (1976) 635-645.

[7] N. Bakhvalov and G. Panasenko, Homogenization: Averaging processes in periodic media. Mathematics and its Applications 36, Kluwer Academic Publishers, Dordrecht (1990).

[8] A. Bensoussan, J.L. Lions and G. Papanicolaou, Asymptotic analysis for periodic structures. North-Holland, Amsterdam (1978).

[9] A. Bensoussan, J.L. Lions and G. Papanicolau, Boundary layer analysis in homogenization of diffusion equations with Dirichlet conditions on the half space, in Proc. Internat. Symposium SDE, K. Ito Ed. J. Wiley, New York (1978) 21-40.

[10] F. Blanc and S.A. Nazarov, Asymptotics of solutions to the Poisson problem in a perforated domain with corners. J. Math. Pures Appl. 76 (1997) 893-911.

[11] D. Gilbarg and N.S. Trudinger, Elliptic partial differential equations of second order. Springer-Verlag, Berlin, Heidelberg, New York (1983).

[12] W. Jäger and A. Mikelic, On the boundary conditions at the contact interface between a porous medium and a free fluid. Ann. Sci. Norm. Sup. Pisa, Serie IV 23 (1996) 404-465.

[13] V.V. Jikov, S.M. Kozlov and O.A. Oleinik, Homogenization of differential operators and integral functionals. Springer-Verlag, Berlin Heidelberg, New York (1994).

[14] J.L. Lions, Some methods in mathematical analysis of systems and their Control. Science Press, Beijing, Gordon and Breach, New York (1981).

[15] S. Moskow and M. Vogelius, First-order corrections to the homogenised eigenvalues of a periodic composite medium. A convergence proof, in Proc. Roy. Soc. Edinburgh., Sect A 127 6 (1997) 1263-1299.

[16] N. Neuss, W. Jäger and G. Wittum, Homogenization and Multigrid. Preprint 1998-04, SFB 359, University of Heidelberg (1998).

[17] M. Neuss-Radu, A result on the decay of the boundary layers in the homogenization theory. Asympto. Anal. 23 (2000) 313-328.

[18] G. Nguetseng, A general convergence result for a functional related to the theory of homogenization. SIAM J. Math. Anal. 20 (1989) 608-623.

[19] O.A. Oleinik, A.S. Shamaev and G.A. Yosifian, Mathematical problems in elasticity and Homogenization. Studies in Mathematics and its Applications 26, North-Holland, Amsterdam (1992).

[20] J. Sanchez-Huber and E. Sanchez-Palencia, Exercices sur les méthodes asymptotiques et l'homogénéisation. Masson, Paris (1993).

[21] E. Sanchez-Palencia, Non-homogenous media and vibration theory. Lect. Notes Phys. 127, Springer-Verlag, Berlin (1980).

[22] J. Wloka, Partielle differentialgleichungen. Teubner-Verlag, Stuttgart (1982).

To access this journal online:

www.edpsciences.org 